Research Article

\title{
The Classification, Automorphism Group, and Derivation Algebra of the Loop Algebra Related to the Nappi-Witten Lie Algebra
}

\author{
Xue Chen \\ School of Applied Mathematics, Xiamen University of Technology, Xiamen 361024, China \\ Correspondence should be addressed to Xue Chen; xuechen@xmut.edu.cn
}

Received 25 May 2021; Accepted 15 July 2021; Published 13 August 2021

Academic Editor: Naihuan Jing

Copyright (c) 2021 Xue Chen. This is an open access article distributed under the Creative Commons Attribution License, which permits unrestricted use, distribution, and reproduction in any medium, provided the original work is properly cited.

Set $L:=H_{4} \otimes_{\mathbb{C}} R, R:=\mathbb{C}\left[t^{ \pm 1}\right]$, and $S:=\mathbb{C}\left[t^{ \pm 1 / m}\right]\left(m \in \mathbb{Z}_{+}\right)$. Then, $L$ is called the loop Nappi-Witten Lie algebra. $R$-isomorphism classes of $S / R$ forms of $L$ are classified. The automorphism group and the derivation algebra of $L$ are also characterized.

\section{Introduction}

The conformal field theory (CFT) plays a significant role in many different areas of mathematics and physics. Wess-Zumino-Novikov-Witten (WZNW) models [1] provide interesting examples of CFTs. WZNW models were first studied in the setting of semisimple groups. Later, since the WZNW models based on non-abelian nonsemisimple Lie groups were found to be closely related to the construction of exact string backgrounds, the special types received some attention $[2,3]$. Nappi and Witten showed in [3] that the WZNW model (NW model) based on a central extension of the twodimensional Euclidean group describes the homogeneous fourdimensional space-time corresponding to a gravitational plane wave. The related Lie algebra is called the Nappi-Witten Lie algebra, which is neither abelian nor semisimple. More results on the NW model were given in [4-7].

The Nappi-Witten Lie algebra $\mathrm{H}_{4}$ is a four-dimensional Lie algebra over $\mathbb{C}$ generated by $\{a, b, c, d\}$ with the following Lie brackets:

$$
\begin{aligned}
{[a, b] } & =c, \\
{[d, a] } & =a, \\
{[d, b] } & =-b, \\
{\left[c, H_{4}\right] } & =0 .
\end{aligned}
$$

There is a nondegenerate invariant symmetric bilinear form (,) on $\mathrm{H}_{4}$ defined by

$$
\begin{aligned}
& (a, b)=1, \\
& (c, d)=1, \quad \text { otherwise, }(,)=0 .
\end{aligned}
$$

Set

$$
\begin{aligned}
L & :=H_{4} \otimes_{\mathbb{C}} R, \\
R & :=\mathbb{C}\left[t^{ \pm 1}\right], \\
S & :=\mathbb{C}\left[t^{ \pm 1 / m}\right], \quad\left(m \in \mathbb{Z}_{+}\right) .
\end{aligned}
$$
follows:

Then, $L$ is a Lie algebra over $\mathbb{C}$ under the bracket as

$$
\left[x \otimes t^{n_{1}}, y \otimes t^{n_{2}}\right]=[x, y] \otimes t^{n_{1}+n_{2}}, \quad \text { for } x, y \in H_{4}, n_{1}, n_{2} \in \mathbb{Z} .
$$

We call $L$ the loop Nappi-Witten Lie algebra. Recently, the representation theory of Lie algebras related to $L$ was studied in $[8,9]$.

Reference [10] studied the automorphism groups of vertex operator algebras associated with the affine Nappi-Witten algebra $\widehat{H}_{4}$. The isomorphism of loop algebras was considered in [11] from the perspective of non-abelian Galois cohomology. Reference [12] gave an explicit description of the algebra of derivations for a large class of 
algebras defined by étale descent. Motivated by the works mentioned above, we study $R$-isomorphism classes of $S / R$ forms of $L$, the automorphism group, and the derivation algebra of $L$ in this paper.

To classify $R$-isomorphism classes of $S / R$ forms of $L$ in Section 2, we recall the results of automorphisms of $H_{4}$ in [10]. The following Lemma 1, Theorem 1, Lemma 2, and Lemma 3 will be used in the proof of Theorem 2 .

Lemma 1 (see [10]). Let $\theta \in \operatorname{Aut}\left(H_{4}\right)$. Then,

$$
\theta(a, b, c, d)=(a, b, c, d)\left(\begin{array}{cccc}
s & 0 & 0 & -s \beta \\
0 & s^{-1} & 0 & -s^{-1} \alpha \\
\alpha & \beta & 1 & -\alpha \beta \\
0 & 0 & 0 & 1
\end{array}\right)
$$

or

$$
\theta(a, b, c, d)=(a, b, c, d)\left(\begin{array}{cccc}
0 & n & 0 & n \xi \\
n^{-1} & 0 & 0 & n^{-1} \rho \\
\xi & \rho & -1 & \xi \rho \\
0 & 0 & 0 & -1
\end{array}\right)
$$

where $s, n \in \mathbb{C}^{*}, \alpha, \beta, \xi, \rho \in \mathbb{C}$.

Define a linear map $\tau$ of $H_{4}$ by

$$
\begin{aligned}
& \tau(a)=b, \\
& \tau(b)=a, \\
& \tau(c)=-c, \\
& \tau(d)=-d .
\end{aligned}
$$

It is clear that $\tau^{2}=\mathrm{id}_{H_{4}}=e$ and $\tau \in \operatorname{Aut}\left(H_{4}\right)$. Set $G_{0}:=\{\tau, e\}$. Then, $G_{0} \cong \mathbb{Z} / 2 \mathbb{Z}$. Set

$$
\begin{aligned}
G_{1} & :=\mathbb{C}^{*} \times(\mathbb{C} \times \mathbb{C}), \\
G & :=\left(\mathbb{C}^{*} \times(\mathbb{C} \times \mathbb{C})\right) \times \frac{\mathbb{Z}}{2 \mathbb{Z}} .
\end{aligned}
$$

The multiplication in group $G$ is defined as follows:

$$
\begin{aligned}
(s, \alpha, \beta, e) \cdot\left(s^{\prime}, \alpha^{\prime}, \beta^{\prime}, e\right) & =\left(s s^{\prime}, \alpha s^{\prime}+\alpha^{\prime}, \beta\left(s^{\prime}\right)^{-1}+\beta^{\prime}, e\right), \\
(n, \xi, \rho, \tau) \cdot\left(n^{\prime}, \xi^{\prime}, \rho^{\prime}, \tau\right) & =\left(n\left(n^{\prime}\right)^{-1}, \rho\left(n^{\prime}\right)^{-1}-\xi^{\prime}, \xi\left(n^{\prime}\right)-\rho^{\prime}, e\right), \\
(n, \xi, \rho, \tau) \cdot(s, \alpha, \beta, e) & =\left(n(s)^{-1}, \xi s-\alpha, \rho s^{-1}-\beta, \tau\right), \\
(s, \alpha, \beta, e) \cdot(n, \xi, \rho, \tau) & =\left(s n, \beta n^{-1}+\xi, n \alpha+\rho, \tau\right),
\end{aligned}
$$

where $s, s^{\prime}, n, n^{\prime} \in \mathbb{C}^{*}, \alpha, \beta, \alpha^{\prime}, \beta^{\prime}, \xi, \rho, \xi^{\prime}, \rho^{\prime} \in \mathbb{C}$. The identity element is $(1,0,0, e)$, and

$$
\begin{aligned}
& (s, \alpha, \beta, e)^{-1}=\left(s^{-1},-\alpha s^{-1},-\beta s, e\right), \\
& (n, \xi, \rho, \tau)^{-1}=\left(n, \rho n^{-1}, \xi n, \tau\right) .
\end{aligned}
$$

Since

$$
\begin{aligned}
& (s, 0,0, e) \cdot(1, \alpha, \beta, e) \cdot(s, 0,0, e)^{-1}=\left(1, \alpha s^{-1}, \beta s, e\right), \\
& (1,0,0, \tau) \cdot(s, \alpha, \beta, e) \cdot(1,0,0, \tau)^{-1}=\left(s^{-1},-\beta,-\alpha, e\right),
\end{aligned}
$$

$\mathbb{C} \times \mathbb{C}$ is a normal subgroup of $G_{1}$ and $G_{1}$ is a normal subgroup of $G$. Thus,

$$
\begin{aligned}
G_{1} & =\mathbb{C}^{*} \ltimes(\mathbb{C} \times \mathbb{C}), \\
G & =\left(\mathbb{C}^{*} \ltimes(\mathbb{C} \times \mathbb{C})\right) \rtimes \frac{\mathbb{Z}}{2 \mathbb{Z}} .
\end{aligned}
$$

Theorem 1 (see [10]). Aut $\left(H_{4}\right) \cong G=\left(\mathbb{C}^{*} \ltimes(\mathbb{C} \times \mathbb{C})\right) \rtimes \mathbb{Z} /$ $2 \mathbb{Z}$.

By Theorem 1, any element of $G$ can be viewed as an automorphism of $\mathrm{H}_{4}$.

Lemma 2. Let $\phi=(s, \alpha, \beta, e)$ and $\psi=(n, \xi, \rho, \tau)$. Then,

$$
\begin{aligned}
\phi^{k} & =\left(s^{k}, \alpha\left(s^{k-1}+s^{k-2}+\ldots+s+1\right), \beta\left(s^{-(k-1)}+s^{-(k-2)}+\ldots+s^{-1}+1\right), e\right), \\
\psi^{2 k} & =\left(1, k\left(\rho n^{-1}-\xi\right), k(\xi n-\rho), e\right), \\
\psi^{2 k+1} & =\left(n,(k+1) \xi-k \rho n^{-1},(k+1) \rho-k n \xi, \tau\right),
\end{aligned}
$$

where $s, n \in \mathbb{C}^{*}, \alpha, \beta, \xi, \rho \in \mathbb{C}$, and $k \in \mathbb{Z}_{+}$.

Proof. It follows from (8) immediately.

\section{Lemma 3}

(1) If $(n, \xi, \rho, \tau)^{2 k}=(1,0,0, e)$ for $k \in \mathbb{Z}_{+}$, then $(n, \xi, \rho, \tau)$ is conjugate to $(1,0,0, \tau)$ in Aut $\left(H_{4}\right)$.
(2) Let $(s, \alpha, \beta, e) \neq(s, 0,0, e)$. Then, $(s, \alpha, \beta, e)$ is conjugate to $(s, 0,0, e)$ in Aut $\left(H_{4}\right)$ if and only if $s \neq 1$.

(3) Let $s, s^{\prime} \neq 1$ and $s \neq s^{\prime}$. Then, $(s, 0,0, e)$ is conjugate to $\left(s^{\prime}, 0,0, e\right)$ in Aut $\left(H_{4}\right)$ if and only if $s s^{\prime}=1$.

Proof

(1) If $(n, \xi, \rho, \tau)^{2 k}=\left(1, k\left(\rho n^{-1}-\quad \xi\right), k(\xi n-\rho), e\right)=$ $(1,0,0, e)$, then $\rho=\xi n$. Choose $(s, \alpha, \beta, e)$ such that $s^{2}=1 / n$ and $n \alpha+\beta=-\rho$. Then, 


$$
(s, \alpha, \beta, e)(n, \xi, \rho, \tau)(s, \alpha, \beta, e)^{-1}=\left(s^{2} n,\left(\alpha+\xi+\beta n^{-1}\right) s^{-1},(n \alpha+\rho+\beta) s, \tau\right)=(1,0,0, \tau)
$$

Thus, $(n, \xi, \rho, \tau)$ is conjugate to $(1,0,0, \tau)$ in $\operatorname{Aut}\left(H_{4}\right)$.

(2) We assume that there exists $\left(s^{\prime}, \alpha^{\prime}, \beta^{\prime}, e\right)$ such that

$$
\left(s^{\prime}, \alpha^{\prime}, \beta^{\prime}, e\right)(s, \alpha, \beta, e)\left(s^{\prime}, \alpha^{\prime}, \beta^{\prime}, e\right)^{-1}=(s, 0,0, e) .
$$

By (8) and (9), we have

$$
\begin{aligned}
\alpha^{\prime}(s-1)+\alpha & =0, \\
\beta^{\prime}\left(s^{-1}-1\right)+\beta & =0 .
\end{aligned}
$$

$$
\begin{array}{r}
(n, \xi, \rho, \tau)(s, 0,0, e)(n, \xi, \rho, \tau)^{-1}=\left(s^{-1},\left(s^{-1}-1\right) \rho n^{-1},(s-1) \xi n, e\right), \\
\left(s^{\prime \prime}, \alpha^{\prime \prime}, \beta^{\prime \prime}, e\right)(s, 0,0, e)\left(s^{\prime \prime}, \alpha^{\prime \prime}, \beta^{\prime \prime}, e\right)^{-1}=\left(s,(s-1) \alpha^{\prime \prime} s \prime^{-1},\left(s^{-1}-1\right) \beta^{\prime \prime} s^{\prime \prime}, e\right), \\
\text { is conjugate to }\left(s^{\prime}, 0,0, e\right) \text { if and only } \\
\square:=k\left[t^{ \pm 1}\right], \\
S:=k\left[t^{ \pm 1 / m}\right],
\end{array}
$$

it is clear that $(s, 0,0, e)$ is conjugate to $\left(s^{\prime}, 0,0, e\right)$ if and only if $s s^{\prime}=1$.

The aim of this paper is to study structures of the loop Nappi-Witten Lie algebra $L=H_{4} \otimes_{\mathbb{C}} R$. Some ideas we use come from [10-12]. This paper is organized as follows. In Section 2, we classify $R$-isomorphism classes of $S / R$ forms of $L$ by the first cohomology set. In Section 3, we determine the automorphism group of $L$. Finally, the derivation algebra of $L$ is also characterized in Section 4.

Throughout the paper, the sets of the complex numbers, the nonzero complex numbers, the nonnegative integers, the integers, and the positive integers are denoted by $\mathbb{C}, \mathbb{C}^{*}, \mathbb{N}$, $\mathbb{Z}$, and $\mathbb{Z}_{+}$, respectively.

\section{The First Cohomology \\ Set $H^{1}\left(\Gamma, \operatorname{Aut}_{S}\left(H_{4}(S)\right)\right)$}

We first review some basic notions and assumptions in [11] and then apply them in the class of $H_{4}$.

Let $k$ be an algebraically closed field of characteristic 0 . For each $m \in \mathbb{Z}_{+}$, we choose a primitive $m^{\text {th }}$ root of unity $\zeta_{m}$ such that these primitive roots of unity are compatible in the sense that $\zeta_{l m}^{l}=\zeta_{m}$ for all $l, m \in \mathbb{Z}_{+}$, and fix the sequence $\left\{\zeta_{m}\right\}_{m \in \mathbb{Z}}$ from now on. Suppose that $\mathfrak{g}$ is a Lie algebra over $k$ and that $\sigma$ is an automorphism of $\mathfrak{g}$ of period $m$ (the order of $\sigma$ might not be equal to $m$, but of course it is a divisor of $m$ ).

Let

$$
\Gamma:=\frac{\mathbb{Z}}{m \mathbb{Z}}=\{\bar{i}: i \in \mathbb{Z}\},
$$

be the group of integers modulo $m$, where $\bar{i}$ denotes the congruence class represented by $i$. Let
Suppose $s=1$. Then, $\alpha=\beta=0$ by (15) is a contradiction. Thus, $s \neq 1$. Conversely, if $s \neq 1$, by (15), $\alpha^{\prime}=$ $\alpha /(1-s)$ and $\beta^{\prime}=\beta /\left(1-s^{-1}\right)$. Then, there exists $\left(s^{\prime}, \alpha^{\prime}, \beta^{\prime}, e\right)\left(s^{\prime} \in \mathbb{C}^{*}\right)$ such that (14) holds. So, $(s, \alpha, \beta, e)$ is conjugate to $(s, 0,0, e)$ in $\operatorname{Aut}\left(H_{4}\right)$.

(3) Since

be two algebras of Laurent polynomials over $k$ and $R \subset S$ is a $k$-subalgebra. If we set $z=t^{1 / m}$, then $t=z^{m}$ and

$$
\begin{aligned}
& R=k\left[z^{ \pm m}\right], \\
& S=k\left[z^{ \pm 1}\right] .
\end{aligned}
$$

It is easy to see that $S$ is an $R$-algebra.

There is an isomorphism of $\Gamma$ onto $\operatorname{Aut}_{R}(S)$ (the group of automorphisms of $S$ which fixes $R$ ) such that

$$
\bar{i} \mapsto \underline{i} \text {, and } \underline{i}(z)=\zeta_{m}^{i} z \text {. }
$$

Let $K \in\{R, S\}$. Set

$$
\mathfrak{g}(K):=\mathfrak{g} \otimes_{k} K,
$$

where $\mathfrak{g}(K)$ is a $K$-module with $K$-action given by $f(x \otimes h)=x \otimes f h$ for $x \in \mathfrak{g}$ and $f, h \in K$. Note that $\mathfrak{g}(K)$ is also a Lie algebra over $K$ under the bracket as follows:

$$
[x \otimes f, y \otimes h]=[x, y] \otimes f h, \quad \text { for } x, y \in \mathfrak{g}, f, h \in K .
$$

We now recall the definition of the loop algebra of $\mathfrak{g}$ relative to $\sigma$. Let

$$
\mathfrak{g}_{\bar{i}}=\left\{x \in \mathfrak{g} \mid \sigma(x)=\zeta_{m}^{i} x\right\} .
$$

The loop algebra of $\mathfrak{g}$ relative to $\sigma$ is the subalgebra

$$
L(\mathfrak{g}, \sigma):=\bigoplus_{i \in \mathbb{Z}} \mathfrak{g}_{i} \otimes_{k} z^{i},
$$

of the Lie algebra 


$$
\mathfrak{g}(S)=\mathfrak{g} \otimes_{k} S .
$$

Note that $L(\mathfrak{g}, \sigma)$ is the set of fixed points in $\mathfrak{g}(S)$ of the $R$-algebra automorphism $\sigma \otimes \underline{1}^{-1}$, and then $L(\mathfrak{g}, \sigma)$ is an $R$-subalgebra of $\mathfrak{g}(S)$. Thus, $L(\mathfrak{g}, \sigma)$ is an $R$-algebra and hence also a $k$-algebra. Furthermore, the $R$-isomorphism class of $L(\mathfrak{g}, \sigma)$ does not depend on the choice of the period $m$ for $\sigma$ (see Lemma 2.3 in [11]).

To interpret loop algebras as $S / R$ forms of the $R$-algebra $\mathfrak{g}(R)$, we review the definitions of the $S / R$ form of $\mathfrak{g}(R)$ and a certain (non-abelian) first cohomology set and relations between them.

An $S / R$ form of $\mathfrak{g}(R)$ is a Lie algebra $\mathscr{F}$ over $R$ such that

$$
\mathscr{F} \otimes_{R} S \cong \mathfrak{g}(S)
$$

as Lie $S$-algebras.

We now recall some concepts of a (non-abelian) cohomology set.

Let $G$ be a group. We say that $\Gamma$-acts on $G$ if there is a map $(\bar{i}, x) \mapsto{ }^{\bar{i}} x$ of $\Gamma \times G \longrightarrow G$ satisfying ${ }^{\bar{i}}\left({ }^{\bar{j}} x\right)={ }^{\bar{i}+\bar{j}} x,{ }^{\overline{0}} x=x$, and ${ }^{\bar{i}}(x y)=\left({ }^{\bar{i}} x\right) \cdot\left({ }^{\bar{i}} y\right)$ for $\bar{i}, \bar{j} \in \Gamma, x, y \in G$.

If $\Gamma$ acts on a group $G$, the map $u: \Gamma \longrightarrow G, \bar{i} \mapsto u_{\bar{i}}$ satisfying

$$
u_{\bar{i}+\bar{j}}=u_{\bar{i}}^{\bar{i}} u_{\bar{j}}
$$

is called 1-cocycle, where ${ }^{\bar{i}} u_{-}$denotes the $\Gamma$-action on $G$. Two 1 -cocycles $u, v$ are said to be cohomologous if there exists some $f \in G$ such that

$$
v_{\bar{i}}=f^{-1} u_{\bar{i}}^{\bar{i}} f
$$

for all $\bar{i} \in \Gamma$. It is an equivalence relation. For every 1 -cocycle $u$, we denote by $[u]$ the equivalence class containing $u$. The set of equivalence classes (called cohomology classes) of 1cocycles from $\Gamma$ to $G$ is denoted by $H^{1}(\Gamma, G)$, and we call it the (non-abelian) first cohomology set.

Define an action of $\Gamma$ on $\operatorname{Aut}_{S}(\mathfrak{g}(S))$ by

$$
{ }^{\bar{i}} \tau:=(\mathrm{id} \otimes \underline{i}) \tau(\mathrm{id} \otimes \underline{i})^{-1},
$$

for $\bar{i} \in \Gamma$ and $\tau \in \operatorname{Aut}_{S}(\mathfrak{g}(S))$, where $\operatorname{Aut}_{S}(\mathfrak{g}(S))$ is the group of automorphisms of $\mathfrak{g}(S)$ as a Lie S-algebra.

The following result comes from Proposition 3.4 of [11].

Proposition 1 (see [11]). $R$-isomorphism classes of $S / R$ forms of $\mathfrak{g}(R)$ are in one-to-one correspondence with cohomology classes in $H^{1}\left(\Gamma, \operatorname{Aut}_{S}(\mathfrak{g}(S))\right)$. Explicitly this correspondence is as follows: if $u$ is a 1 -cocycle on $\Gamma$ in $\operatorname{Aut}_{S}(\mathfrak{g}(S))$, then the cohomology class $[u]$ corresponds to the $R$-isomorphism classes of the $S / R$ form:

$$
\mathfrak{g}(S)_{u}:=\left\{x \in \mathfrak{g}(S):\left(u_{\bar{i}}(\mathrm{id} \otimes \underline{i})\right) x=x \text { for } \bar{i} \in \Gamma\right\},
$$

of $\mathfrak{g}(S)$.

Furthermore, Theorem 3.6 in [11] gives our description of loop algebras as $S / R$ forms of $\mathfrak{g}(R)$. In view of Theorem 3.6 in [11], the loop algebra $L(\mathfrak{g}, \sigma)$ is an $S / R$-form of $\mathfrak{g}(R)$ and is determined by the 1-cocycle:

$$
\begin{aligned}
u: \Gamma \longrightarrow \operatorname{Aut}_{S}(\mathfrak{g}(S)), & \\
\bar{i} & \longmapsto \sigma^{-i} \otimes \mathrm{id} .
\end{aligned}
$$

So, we can write $L(\mathfrak{g}, \sigma)=\mathfrak{g}(S)_{\mathcal{u}}$, where

$$
u_{\bar{i}}=\sigma^{-i} \otimes \mathrm{id}, \quad \text { for } \bar{i} \in \Gamma \text {. }
$$

In the following, set $k=\mathbb{C}$ and $\mathfrak{g}=H_{4}$. Let $S$ and $R$ be the algebras of Laurent polynomials over $\mathbb{C}$ as in (19). We will classify loop algebras of $H_{4}$ from the view of $S / R$ forms of $H_{4}(R)$.

Lemma 4. Let $\sigma, \tau \in A u t\left(H_{4}\right)$ and $\sigma^{m}=\tau^{m}=i d_{H_{4}}$. We define 1-cocycles $u$ and $v$ on $\Gamma$ in $A u t_{S}\left(H_{4}(S)\right)$ by

$$
u_{\bar{i}}=\sigma^{-i} \otimes \mathrm{id} \text {, and } v_{\bar{i}}=\tau^{-i} \otimes \mathrm{id}, \forall \bar{i} \in \Gamma \text {. }
$$

If $\sigma$ is conjugate to $\tau$ in Aut $\left(H_{4}\right)$, then $[u]=[v]$.

Proof. Suppose that there exists $h \in \operatorname{Aut}\left(H_{4}\right)$ such that $h^{-1} \sigma h=\tau$. Then, $h^{-1} \sigma^{-i} h=\tau^{-i}, \forall i \in \mathbb{Z}$. Set $f=h \otimes \mathrm{id}$, then $f \in \operatorname{Aut}_{S}\left(H_{4}(S)\right)$. For any $\bar{i} \in \Gamma$ and $x \otimes z^{n} \in H_{4}(S)$, we have

$$
\begin{aligned}
f^{-1} u_{\bar{i}}^{\bar{i}} f\left(x \otimes z^{n}\right) & =\left(h^{-1} \otimes \mathrm{id}\right)\left(\sigma^{-i} \otimes \mathrm{id}\right)(\mathrm{id} \otimes \underline{i})(h \otimes \mathrm{id})(\mathrm{id} \otimes \underline{i})^{-1}\left(x \otimes z^{n}\right) \\
& =h^{-1} \sigma^{-i} h(x) \otimes z^{n} \\
& =\tau^{-i}(x) \otimes z^{n} \\
& =v_{\bar{i}}\left(x \otimes z^{n}\right) .
\end{aligned}
$$

Thus, $[u]=[v]$.

$\left\{\phi_{m, r}:=\left(\zeta_{m}^{r}, 0,0, e\right) \mid 0 \leq r \leq\left[\frac{m}{2}\right]\right\}, \quad \psi:=(1,0,0, \tau)$

Theorem 2. Let $\Gamma=\mathbb{Z} / m \mathbb{Z}$.

of period $m$ of $\mathrm{H}_{4}$ in the sense of conjugation. Furthermore, if $m \in 2 \mathbb{Z}+1, \psi$ does not exist.

(i) Then, there are automorphisms

(ii) If we define 1-cocycles $u_{m, r}$, v on $\Gamma$ in Aut $t_{S}\left(H_{4}(S)\right)$ by 


$$
\begin{aligned}
\left(u_{m, r}\right)_{\bar{i}} & =\phi_{m, r}^{-i} \otimes \mathrm{id}, \\
v_{\bar{i}} & =\psi^{-i} \otimes \mathrm{id}, \quad \forall \bar{i} \in \Gamma, 0 \leq r \leq\left[\frac{m}{2}\right],
\end{aligned}
$$

then the corresponding $R$-isomorphism classes of the $S / R$ form of $H_{4}(R)$ are as follows:

$$
\begin{aligned}
H_{4}(S)_{u_{m, r}} & =\operatorname{Span}_{\mathbb{C}}\left\{a \otimes z^{m k+r}, b \otimes z^{m k+(m-r)}, c \otimes z^{m k}, d \otimes z^{m k} \mid k \in \mathbb{Z}, \quad 0 \leq r \leq\left[\frac{m}{2}\right]\right\}, \\
H_{4}(S)_{v} & =\operatorname{Span}_{\mathbb{C}}\left\{(a-b) \otimes z^{2 k+1}, c \otimes z^{2 k+1}, d \otimes z^{2 k+1},(a+b) \otimes z^{2 k} \mid k \in \mathbb{Z}\right\} .
\end{aligned}
$$

Furthermore, if $m \in 2 \mathbb{Z}+1$, neither $v$ nor $H_{4}(S)_{v}$ exists.

Proof (i) Let $\psi=(n, \xi, \rho, \tau) \in \operatorname{Aut}\left(H_{4}\right)$ such that $\psi^{m}=$ $(1,0,0, e)$. Then, $m \in 2 \mathbb{Z}_{+}$by Lemma 2 , and $\psi$ is conjugate to $(1,0,0, \tau)$ in Aut $\left(H_{4}\right)$ by Lemma 3(1). Let $\varphi=(s, \alpha, \beta, e) \in \operatorname{Aut}\left(H_{4}\right)$ such that

$$
\begin{aligned}
\varphi^{m} & =\left(s^{m}, \alpha\left(s^{m-1}+s^{m-2}+\ldots+s+1\right), \beta\left(s^{-(m-1)}+s^{-(m-2)}+\ldots+s^{-1}+1\right), e\right) \\
& =(1,0,0, e) .
\end{aligned}
$$

Then,

$$
\begin{aligned}
\varphi_{m, r} & =\left(\zeta_{m}^{r}, \alpha, \beta, e\right), \quad(1 \leq r \leq m-1), \\
\varphi_{m, 0} & =(1,0,0, e) .
\end{aligned}
$$

For any $1 \leq r \leq m-1, \varphi_{m, r}=\left(\zeta_{m}^{r}, \alpha, \beta, e\right)$ is conjugate to $\phi_{m, r}=\left(\zeta_{m}^{r}, 0,0, e\right)$ by Lemma 3(2). Furthermore, $\left\{\phi_{m, r}=\left(\zeta_{m}^{r}, 0,0, e\right) \mid 0 \leq r \leq[m / 2]\right\}$ are not conjugate with each other by Lemma 3(3) and clearly not conjugate to $(1,0,0, \tau)$.

(ii) It follows from Proposition 1 and Lemma 4 by straightforward computation.

\section{The Automorphism Group $\operatorname{Aut}_{\mathbb{C}}(L)$ of $L$}

In this section, we study the automorphisms of $L$, where $L=H_{4} \otimes_{\mathbb{C}} R, R=\mathbb{C}\left[t^{ \pm 1}\right]$. Let $\operatorname{Aut}_{R}(L)$ denote the group of all $R$-linear automorphisms of $L$.

We first recall the definition of the centroid in [11] that will be used later. Let $K$ be a unital commutative associative algebra over $\mathbb{C}$ and $T$ be a Lie algebra over $K$. Set

$$
\begin{aligned}
\operatorname{Ctd}_{K}(T) & =\left\{\chi \in \operatorname{End}_{K-\bmod }(T) \mid \chi([x, y])\right. \\
& =[x, \chi(y)] \text { for all } x, y \in T\},
\end{aligned}
$$

where $\operatorname{Ctd}_{K}(T)$ is called the centroid of $T$ over $K$. Define $\lambda_{T}: K \longrightarrow \operatorname{End}_{K-\text { mod }}(T)$ by

$$
\lambda_{T}(r)(x)=r x, \quad \text { for } r \in K \text { and } x \in T .
$$

It is clear that $\lambda_{T}(K) \subseteq \operatorname{Ctd}_{K}(T)$. We say that $T$ is central over $K$ if $\operatorname{Ctd}_{K}(T)=\lambda_{T}(K)$ (see [11]).

Lemma 5. Let $\chi \in \operatorname{Ctd}_{\mathbb{C}}\left(H_{4}\right)$. Then,

$$
\begin{aligned}
\chi(x) & =\lambda_{1} x, \\
\chi(d) & =\lambda_{1} d+\rho c, \\
x & \in\{a, b, c\}, \quad \lambda_{1}, \rho \in \mathbb{C} .
\end{aligned}
$$

Proof. Let $\chi \in \operatorname{Ctd}_{\mathbb{C}}\left(H_{4}\right)$. We can assume that

$$
\begin{aligned}
& \chi(a)=\lambda_{1} a+\mu_{1} b, \\
& \chi(b)=\lambda_{2} a+\mu_{2} b,
\end{aligned}
$$

where $\lambda_{i}, \mu_{i} \in \mathbb{C}, i=1,2$. Since

$$
\begin{aligned}
\chi(a)=\chi([d, a]) & =[d, \chi(a)]=[\chi(d), a], \\
-\chi(b)=\chi([d, b]) & =[d, \chi(b)]=[\chi(d), b], \\
\chi(c)=\chi([a, b]) & =[a, \chi(b)]=[\chi(a), b],
\end{aligned}
$$

we have $\mu_{1}=\lambda_{2}=0$ and $\lambda_{1}=\mu_{2}$. Thus,

$$
\begin{aligned}
& \chi(a)=\lambda_{1} a, \\
& \chi(b)=\lambda_{1} b, \\
& \chi(c)=\lambda_{1} c, \\
& \chi(d)=\lambda_{1} d+\rho c, \quad \lambda_{1}, \rho \in \mathbb{C} .
\end{aligned}
$$

Theorem 3. If $\chi \in C t d_{\mathbb{C}}(L)$, then

$$
\begin{aligned}
& \chi\left(x \otimes t^{n}\right)=\lambda_{0}^{\chi}(t)\left(x \otimes t^{n}\right), \\
& \chi\left(d \otimes t^{n}\right)=\lambda_{0}^{\chi}(t)\left(d \otimes t^{n}\right)+\alpha_{n}^{\chi}(t)\left(c \otimes t^{0}\right),
\end{aligned}
$$

where $x \in\{a, b, c\}, \lambda_{0}^{\chi}(t), \alpha_{n}^{\chi}(t) \in R$, and $n \in \mathbb{Z}$.

Proof. We assume that 


$$
\begin{aligned}
& \chi\left(a \otimes t^{n}\right)=\lambda_{n}^{\chi}(t)\left(a \otimes t^{0}\right)+\mu_{n}^{\chi}(t)\left(b \otimes t^{0}\right), \\
& \chi\left(b \otimes t^{n}\right)=\delta_{n}^{\chi}(t)\left(a \otimes t^{0}\right)+\rho_{n}^{\chi}(t)\left(b \otimes t^{0}\right),
\end{aligned}
$$

where $\lambda_{n}^{\chi}(t), \mu_{n}^{\chi}(t), \delta_{n}^{\chi}(t), \rho_{n}^{\chi}(t) \in R$. By

$$
\begin{aligned}
& {\left[d \otimes t^{0}, \chi\left(a \otimes t^{n}\right)\right]=\chi\left(a \otimes t^{n}\right)=\left[d \otimes t^{n}, \chi\left(a \otimes t^{0}\right)\right]=\left[\chi\left(d \otimes t^{n}\right), a \otimes t^{0}\right]} \\
& {\left[d \otimes t^{0}, \chi\left(b \otimes t^{n}\right)\right]=-\chi\left(b \otimes t^{n}\right)=\left[d \otimes t^{n}, \chi\left(b \otimes t^{0}\right)\right]=\left[\chi\left(d \otimes t^{n}\right), b \otimes t^{0}\right],} \\
& {\left[\chi\left(a \otimes t^{n}\right), b \otimes t^{0}\right]=\chi\left(c \otimes t^{n}\right)=\left[a \otimes t^{n}, \chi\left(b \otimes t^{0}\right)\right],}
\end{aligned}
$$

we have

$$
\mu_{n}^{\chi}(t)=\delta_{n}^{\chi}(t)=0, \lambda_{n}^{\chi}(t)=\lambda_{0}^{\chi}(t) t^{n}, \rho_{n}^{\chi}(t)=\rho_{0}^{\chi}(t) t^{n}, \lambda_{0}^{\chi}(t)=\rho_{0}^{\chi}(t) .
$$

So,

$$
\begin{aligned}
& \chi\left(a \otimes t^{n}\right)=\lambda_{0}^{\chi}(t)\left(a \otimes t^{n}\right), \\
& \chi\left(b \otimes t^{n}\right)=\lambda_{0}^{\chi}(t)\left(b \otimes t^{n}\right), \\
& \chi\left(c \otimes t^{n}\right)=\lambda_{0}^{\chi}(t)\left(c \otimes t^{n}\right), \\
& \chi\left(d \otimes t^{n}\right)=\lambda_{0}^{\chi}(t)\left(d \otimes t^{n}\right)+\alpha_{n}^{\chi}(t)\left(c \otimes t^{0}\right), \quad \lambda_{0}^{\chi}(t), \alpha_{n}^{\chi}(t) \in R .
\end{aligned}
$$

Corollary 1. $C t d_{\mathbb{C}}(L) \cong R \times \operatorname{Hom}(L /[L, L], Z(L))$ as vector spaces over $\mathbb{C}$, where $Z(L)$ is the center of $L$.

Proof. Define the map

$$
\begin{aligned}
\tau: \operatorname{Ctd}_{\mathbb{C}}(L) & \longrightarrow R \times \operatorname{Hom}\left(\frac{L}{[L, L]}, Z(L)\right), \\
\chi & \mapsto\left(\lambda_{0}^{\chi}(t), \varphi^{\chi}\right),
\end{aligned}
$$

and $\varphi^{\chi}\left(d \otimes t^{n}\right)=\alpha_{n}^{\chi}(t)\left(c \otimes t^{0}\right)$ for any $n \in \mathbb{Z}$. It is easy to see that $\tau$ is a vector space isomorphism.

Corollary 2. If $\chi \in C t d_{R}(L)$, then

$$
\begin{aligned}
& \chi\left(x \otimes t^{n}\right)=\lambda_{0}^{\chi}(t)\left(x \otimes t^{n}\right), \\
& \chi\left(d \otimes t^{n}\right)=\lambda_{0}^{\chi}(t)\left(d \otimes t^{n}\right)+\alpha_{0}^{\chi}(t)\left(c \otimes t^{n}\right),
\end{aligned}
$$

where $x \in\{a, b, c\}, \quad \lambda_{0}^{\chi}(t), \quad \alpha_{0}^{\chi}(t) \in R, \quad$ and $C t d_{R}(L)$ is commutative.

Proof. We just need to prove the commutativity of $\operatorname{Ctd}_{R}(L)$. Let $\chi_{1}, \chi_{2} \in \operatorname{Ctd}_{R}(L)$. By Theorem 3, it is clear that

$$
\begin{aligned}
\chi_{1} \chi_{2}\left(x \otimes t^{n}\right) & =\chi_{1}\left(\lambda_{0}^{\chi_{2}}(t) x \otimes t^{n}\right)=\lambda_{0}^{\chi_{2}}(t) \lambda_{0}^{\chi_{1}}(t)\left(x \otimes t^{n}\right) \\
& =\chi_{2} \chi_{1}\left(x \otimes t^{n}\right),
\end{aligned}
$$

for $x \in\{a, b, c\}$ and $n \in \mathbb{Z}$. Moreover,

$$
\begin{aligned}
\chi_{1} \chi_{2}\left(d \otimes t^{n}\right) & =\chi_{1}\left[\lambda_{0}^{\chi_{2}}(t)\left(d \otimes t^{n}\right)+\alpha_{0}^{\chi_{2}}(t)\left(c \otimes t^{n}\right)\right] \\
& =\lambda_{0}^{\chi_{2}}(t)\left[\lambda_{0}^{\chi_{1}}(t)\left(d \otimes t^{n}\right)+\alpha_{0}^{\chi_{1}}(t)\left(c \otimes t^{n}\right)\right]+\alpha_{0}^{\chi_{2}}(t) \lambda_{0}^{\chi_{1}}(t)\left(c \otimes t^{n}\right) \\
& =\lambda_{0}^{\chi_{1}}(t) \lambda_{0}^{\chi_{2}}(t)\left(d \otimes t^{n}\right)+\left[\alpha_{0}^{\chi_{1}}(t) \lambda_{0}^{\chi_{2}}(t)+\alpha_{0}^{\chi_{2}}(t) \lambda_{0}^{\chi_{1}}(t)\right]\left(c \otimes t^{n}\right) \\
& =\chi_{2} \chi_{1}\left(d \otimes t^{n}\right) .
\end{aligned}
$$

Corollary 3. $\operatorname{Ctd}_{R}(L)=C t d_{R}\left(H_{4} \otimes_{\mathbb{C}} R\right) \cong C t d_{\mathbb{C}}\left(H_{4}\right) \otimes_{\mathbb{C}} R$ as algebras over $R$.

Proof. By Lemma 5, we can define $\varphi, \psi \in \operatorname{Ctd}_{\mathbb{C}}\left(H_{4}\right)$ by

$$
\begin{aligned}
& \varphi(x)=\lambda x, x \in\{a, b, c, d\}, \\
& \psi(y)=0, \quad y \in\{a, b, c\}, \\
& \psi(d)=\rho c, \quad \lambda, \rho \in \mathbb{C} .
\end{aligned}
$$

Define the map

$$
\begin{aligned}
& \eta: \operatorname{Ctd}_{R}(L) \longrightarrow \operatorname{Ctd}_{\mathbb{C}}\left(H_{4}\right) \otimes_{\mathbb{C}} R, \\
& \chi \mapsto \varphi \otimes \frac{\lambda_{0}^{\chi}(t)}{\lambda}+\psi \otimes \frac{\alpha_{0}^{\chi}(t)}{\rho},
\end{aligned}
$$

where $\lambda, \rho \in \mathbb{C}^{*}, \lambda_{0}^{\chi}(t), \alpha_{0}^{\chi}(t) \in R$. It is easy to check that $\eta$ is an $R$-algebra isomorphism. 
Remark 1. Corollary 3 can also follow from Lemma 5 in [12].

Lemma 6. If $\theta \in A u t_{\mathbb{C}}(L)$, then

$$
\begin{aligned}
& \theta\left(x \otimes t^{k}\right)=\lambda_{0} \lambda_{k}(t) \theta\left(x \otimes t^{0}\right), \quad x \in\{a, b, c\}, \\
& \theta\left(d \otimes t^{k}\right)=\lambda_{0} \lambda_{k}(t) \theta\left(d \otimes t^{0}\right)+\left(\delta_{k}(t)-\lambda_{0} \lambda_{k}(t) \delta_{0}(t)\right)\left(c \otimes t^{0}\right),
\end{aligned}
$$

where $\lambda_{k}(t), \delta_{k}(t) \in R, k \in \mathbb{Z}$, and $\lambda_{k_{1}+k_{2}}(t)=\lambda_{0} \lambda_{k_{1}}(t) \lambda_{k_{2}}(t)$, $\lambda_{0}:=\lambda_{0}(t) \in\{ \pm 1\}$.

Proof. Let $\theta \in \operatorname{Aut}_{\mathbb{C}}(L)$. We can assume that

$$
\begin{aligned}
\theta\left(a \otimes t^{k}\right)= & s_{k}(t)\left(a \otimes t^{0}\right)+m_{k}(t)\left(b \otimes t^{0}\right)+\alpha_{k}(t)\left(c \otimes t^{0}\right), \\
\theta\left(b \otimes t^{k}\right)= & n_{k}(t)\left(a \otimes t^{0}\right)+l_{k}(t)\left(b \otimes t^{0}\right)+\beta_{k}(t)\left(c \otimes t^{0}\right), \\
\theta\left(d \otimes t^{k}\right)= & \lambda_{k}(t)\left(d \otimes t^{0}\right)+\mu_{k}^{1}(t)\left(a \otimes t^{0}\right)+\mu_{k}^{2}(t)\left(b \otimes t^{0}\right) \\
& +\delta_{k}(t)\left(c \otimes t^{0}\right),
\end{aligned}
$$

where $s_{k}(t), m_{k}(t), \alpha_{k}(t), n_{k}(t), l_{k}(t), \beta_{k}(t), \lambda_{k}(t), \mu_{k}^{1}(t)$, $\mu_{k}^{2}(t), \delta_{k}(t) \in R$. By

$$
\begin{aligned}
& {\left[\theta\left(d \otimes t^{0}\right), \theta\left(a \otimes t^{k}\right)\right]=\theta\left(a \otimes t^{k}\right)=\left[\theta\left(d \otimes t^{k}\right), \theta\left(a \otimes t^{0}\right)\right],} \\
& {\left[\theta\left(d \otimes t^{0}\right), \theta\left(b \otimes t^{k}\right)\right]=-\theta\left(b \otimes t^{k}\right)=\left[\theta\left(d \otimes t^{k}\right), \theta\left(b \otimes t^{0}\right)\right],}
\end{aligned}
$$

we deduce that

$$
\begin{aligned}
\lambda_{0}(t) & =1, \\
m_{k}(t) & =n_{k}(t)=0, \\
s_{k}(t) & =\lambda_{k}(t) s_{0}(t), \\
\alpha_{k}(t) & =\lambda_{k}(t) \alpha_{0}(t), \\
l_{k}(t) & =\lambda_{k}(t) l_{0}(t), \\
\beta_{k}(t) & =\lambda_{k}(t) \beta_{0}(t), \\
\mu_{k}^{1}(t) & =\lambda_{k}(t) \mu_{0}^{1}(t), \\
\mu_{k}^{2}(t) & =\lambda_{k}(t) \mu_{0}^{2}(t),
\end{aligned}
$$

or

$$
\begin{aligned}
\lambda_{0}(t) & =-1 \\
s_{k}(t) & =l_{k}(t)=0 \\
m_{k}(t) & =-\lambda_{k}(t) m_{0}(t) \\
\alpha_{k}(t) & =-\lambda_{k}(t) \alpha_{0}(t), \\
n_{k}(t) & =-\lambda_{k}(t) n_{0}(t) \\
\beta_{k}(t) & =-\lambda_{k}(t) \beta_{0}(t), \\
\mu_{k}^{1}(t) & =-\lambda_{k}(t) \mu_{0}^{1}(t), \\
\mu_{k}^{2}(t) & =-\lambda_{k}(t) \mu_{0}^{2}(t)
\end{aligned}
$$
have

By $(60)$ and $(61)$ and $\theta\left(c \otimes t^{k}\right)=\left[\theta\left(a \otimes t^{k}\right), \theta\left(b \otimes t^{0}\right)\right]$, we

$$
\begin{aligned}
\lambda_{0}(t) & =1, \\
\theta\left(a \otimes t^{k}\right) & =\lambda_{k}(t)\left(s_{0}(t)\left(a \otimes t^{0}\right)+\alpha_{0}(t)\left(c \otimes t^{0}\right)\right)=\lambda_{k}(t) \theta\left(a \otimes t^{0}\right), \\
\theta\left(b \otimes t^{k}\right) & =\lambda_{k}(t)\left(l_{0}(t)\left(b \otimes t^{0}\right)+\beta_{0}(t)\left(c \otimes t^{0}\right)\right)=\lambda_{k}(t) \theta\left(b \otimes t^{0}\right), \\
\theta\left(c \otimes t^{k}\right) & =\lambda_{k}(t) s_{0}(t) l_{0}(t)\left(c \otimes t^{0}\right)=\lambda_{k}(t) \theta\left(c \otimes t^{0}\right), \\
\theta\left(d \otimes t^{k}\right) & =\lambda_{k}(t)\left(d \otimes t^{0}\right)+\lambda_{k}(t) \mu_{0}^{1}(t)\left(a \otimes t^{0}\right)+\lambda_{k}(t) \mu_{0}^{2}(t)\left(b \otimes t^{0}\right)+\delta_{k}(t)\left(c \otimes t^{0}\right) \\
& =\lambda_{k}(t) \theta\left(d \otimes t^{0}\right)+\left(\delta_{k}(t)-\lambda_{k}(t) \delta_{0}(t)\right)\left(c \otimes t^{0}\right),
\end{aligned}
$$

or

$$
\begin{aligned}
\lambda_{0}(t) & =-1, \\
\theta\left(a \otimes t^{k}\right) & =-\lambda_{k}(t)\left(m_{0}(t)\left(b \otimes t^{0}\right)+\alpha_{0}(t)\left(c \otimes t^{0}\right)\right)=-\lambda_{k}(t) \theta\left(a \otimes t^{0}\right), \\
\theta\left(b \otimes t^{k}\right) & =-\lambda_{k}(t)\left(n_{0}(t)\left(a \otimes t^{0}\right)+\beta_{0}(t)\left(c \otimes t^{0}\right)\right)=-\lambda_{k}(t) \theta\left(b \otimes t^{0}\right), \\
\theta\left(c \otimes t^{k}\right) & =\lambda_{k}(t) m_{0}(t) n_{0}(t)\left(c \otimes t^{0}\right)=-\lambda_{k}(t) \theta\left(c \otimes t^{0}\right), \\
\theta\left(d \otimes t^{k}\right) & =\lambda_{k}(t)\left(d \otimes t^{0}\right)-\lambda_{k}(t) \mu_{0}^{1}(t)\left(a \otimes t^{0}\right)-\lambda_{k}(t) \mu_{0}^{2}(t)\left(b \otimes t^{0}\right)+\delta_{k}(t)\left(c \otimes t^{0}\right) \\
& =-\lambda_{k}(t) \theta\left(d \otimes t^{0}\right)+\left(\delta_{k}(t)+\lambda_{k}(t) \delta_{0}(t)\right)\left(c \otimes t^{0}\right) .
\end{aligned}
$$


Since

$$
\begin{aligned}
{\left[\theta\left(d \otimes t^{k_{1}}\right), \theta\left(a \otimes t^{k_{2}}\right)\right] } & =\theta\left(a \otimes t^{k_{1}+k_{2}}\right)=\lambda_{0}(t) \lambda_{k_{1}+k_{2}}(t) \theta\left(a \otimes t^{0}\right) \\
& =\left[\lambda_{0}(t) \lambda_{k_{1}}(t) \theta\left(d \otimes t^{0}\right)+\left(\delta_{k_{1}}(t)-\lambda_{0}(t) \lambda_{k_{1}}(t) \delta_{0}(t)\right)\left(c \otimes t^{0}\right), \lambda_{0}(t) \lambda_{k_{2}}(t) \theta\left(a \otimes t^{0}\right)\right] \\
& =\lambda_{0}^{2}(t) \lambda_{k_{1}}(t) \lambda_{k_{2}}(t) \theta\left(a \otimes t^{0}\right),
\end{aligned}
$$

we have $\lambda_{k_{1}+k_{2}}(t)=\lambda_{0}(t) \lambda_{k_{1}}(t) \lambda_{k_{2}}(t)$. The lemma is proved.

$$
\theta_{c}\left(t^{n}\right)=\lambda_{0} \lambda_{n}(t) \Leftrightarrow \pi\left(\theta\left(t^{n}, 0\right) \theta^{-1}\right)=\lambda_{0} \lambda_{n}(t) .
$$

Define a map $\pi: R \times \operatorname{Hom}(L /[L, L], Z(L)) \longrightarrow R$ by

$$
\pi(r, \varphi)=r
$$

for $\quad r \in R, \quad \varphi \in \operatorname{Hom}(L /[L, L], Z(L))$. Furthermore, $(r, 0)\left(x \otimes t^{k}\right)=x \otimes r t^{k}$ for $x \otimes t^{k} \in L$.

Then, $\theta_{c} \in A u t_{\mathbb{C}}(R)$.

Proof

(1) For any $X, Y \in L$, we have

\section{Lemma 7}

(1) Let $\theta \in A u t_{\mathbb{C}}(L)$. Then, $\theta\left(t^{n}, 0\right) \theta^{-1} \in C t d_{\mathbb{C}}(L)$ and $\pi\left(\theta\left(t^{n}, 0\right) \theta^{-1}\right)=\lambda_{0} \lambda_{n}(t) ;$

(2) Given $\theta \in A u t_{\mathbb{C}}(L)$. The linear map $\wedge: A u t_{\mathbb{C}}(L)$ $\longrightarrow A u t_{\mathbb{C}}(R)$ is defined by the following identity:

$$
\begin{aligned}
\theta\left(t^{n}, 0\right) \theta^{-1}[X, Y] & =\theta\left[\left(t^{n}, 0\right) \theta^{-1} X, \theta^{-1} Y\right] \\
& =\left[\theta\left(t^{n}, 0\right) \theta^{-1} X, Y\right] .
\end{aligned}
$$

Then, $\theta\left(t^{n}, 0\right) \theta^{-1} \in \operatorname{Ctd}_{\mathbb{C}}(L)$. By Lemma 6, for $x \in\{a, b, c\}$, we have

$$
\begin{aligned}
\theta\left(t^{n}, 0\right) \theta^{-1}\left(\theta\left(x \otimes t^{k}\right)\right) & =\theta\left(t^{n}, 0\right)\left(x \otimes t^{k}\right) \\
& =\theta\left(x \otimes t^{n+k}\right) \\
& =\lambda_{0} \lambda_{n+k}(t) \theta\left(x \otimes t^{0}\right) \\
& =\lambda_{0}^{2} \lambda_{n}(t) \lambda_{k}(t) \theta\left(x \otimes t^{0}\right) \\
& =\lambda_{0} \lambda_{n}(t) \theta\left(x \otimes t^{k}\right), \\
\theta\left(t^{n}, 0\right) \theta^{-1}\left(\theta\left(d \otimes t^{k}\right)\right) & =\theta\left(d \otimes t^{n+k}\right) \\
& =\lambda_{0} \lambda_{n+k}(t) \theta\left(d \otimes t^{0}\right)+\left(\delta_{n+k}(t)-\lambda_{0} \lambda_{n+k}(t) \delta_{0}(t)\right)\left(c \otimes t^{0}\right) \\
& =\lambda_{0}^{2} \lambda_{n}(t) \lambda_{k}(t) \theta\left(d \otimes t^{0}\right)+\left(\delta_{n+k}(t)-\lambda_{0}^{2} \lambda_{n}(t) \lambda_{k}(t) \delta_{0}(t)\right)\left(c \otimes t^{0}\right) \\
& =\lambda_{0} \lambda_{n}(t) \theta\left(d \otimes t^{k}\right)+\left(\delta_{n+k}(t)-\lambda_{0} \lambda_{n}(t) \delta(t)\right)\left(c \otimes t^{0}\right) .
\end{aligned}
$$

Then, by (62)-(63) and Theorem 3, we deduce that $\pi\left(\theta\left(t^{n}, 0\right) \theta^{-1}\right)=\lambda_{0} \lambda_{n}(t)$.

(2) By the definition of $\theta_{c}$ and Lemma 6 , for $x \in\{a, b, c\}$, we have

$$
\begin{aligned}
\theta\left(t^{n_{1}}+t^{n_{2}}, 0\right) \theta^{-1}\left(\theta\left(x \otimes t^{k}\right)\right) & =\theta\left(t^{n_{1}}+t^{n_{2}}, 0\right)\left(x \otimes t^{k}\right) \\
& =\theta\left(x \otimes\left(t^{n_{1}+k}+t^{n_{2}+k}\right)\right) \\
& =\lambda_{0}\left(\lambda_{n_{1}}(t)+\lambda_{n_{2}}(t)\right) \theta\left(x \otimes t^{k}\right) \\
& =\left(\theta_{c}\left(t^{n_{1}}\right)+\theta_{c}\left(t^{n_{2}}\right)\right) \theta\left(x \otimes t^{k}\right),
\end{aligned}
$$




$$
\begin{aligned}
\theta\left(t^{n_{1}}+t^{n_{2}}, 0\right) \theta^{-1}\left(\theta\left(d \otimes t^{k}\right)\right)= & \theta\left(d \otimes t^{n_{1}+k}\right)+\theta\left(d \otimes t^{n_{2}+k}\right) \\
= & \lambda_{0} \lambda_{n_{1}}(t) \theta\left(d \otimes t^{k}\right)+\left(\delta_{n_{1}+k}(t)-\lambda_{0} \lambda_{n_{1}}(t) \delta_{k}(t)\right)\left(c \otimes t^{0}\right)+\lambda_{0} \lambda_{n_{2}}(t) \theta\left(d \otimes t^{k}\right) \\
& +\left(\delta_{n_{2}+k}(t)-\lambda_{0} \lambda_{n_{2}}(t) \delta_{k}(t)\right)\left(c \otimes t^{0}\right) \\
= & \left(\theta_{c}\left(t^{n_{1}}\right)+\theta_{c}\left(t^{n_{2}}\right)\right) \theta\left(d \otimes t^{k}\right)+\sum_{i=1}^{2}\left(\delta_{n_{i}+k}(t)-\lambda_{0} \lambda_{n_{i}}(t) \delta_{k}(t)\right)\left(c \otimes t^{0}\right) .
\end{aligned}
$$

Thus, $\theta_{c}\left(t^{n_{1}}+t^{n_{2}}\right)=\theta_{c}\left(t^{n_{1}}\right)+\theta_{c}\left(t^{n_{2}}\right)$. Similarly, we can get $\theta_{c}\left(\mu t^{n}\right)=\mu \theta_{c}\left(t^{n}\right)$ for any $\mu \in \mathbb{C}$. Furthermore, we have $\theta_{c}\left(t^{n_{1}} \cdot t^{n_{2}}\right)=\lambda_{0} \lambda_{n_{1}+n_{2}}(t)=\lambda_{0}^{2} \lambda_{n_{1}}(t) \lambda_{n_{2}}(t)=\theta_{c}\left(t^{n_{1}}\right) \cdot \theta_{c}\left(t^{n_{2}}\right)$.
In the following, we check that $\left(\theta_{c}\right)^{-1}=\left(\theta^{-1}\right)_{c}$. By $\theta \in \operatorname{Aut}_{\mathbb{C}}(L)$ and Lemma 6 , we can assume that

$$
\begin{aligned}
\theta^{-1}\left(c \otimes t^{k}\right) & =\lambda_{0}^{\prime} \lambda_{k}^{\prime}(t) \theta^{-1}\left(c \otimes t^{0}\right), \\
& = \begin{cases}\lambda_{k}^{\prime}(t) s_{0}^{\prime}(t) l_{0}^{\prime}(t)\left(c \otimes t^{0}\right)=\lambda_{k}^{\prime}(t) \theta^{-1}\left(c \otimes t^{0}\right), & \text { if } \lambda_{0}^{\prime}=1, \\
\lambda_{k}^{\prime}(t) m_{0}^{\prime}(t) n_{0}^{\prime}(t)\left(c \otimes t^{0}\right)=-\lambda_{k}^{\prime}(t) \theta^{-1}\left(c \otimes t^{0}\right), & \text { if } \lambda_{0}^{\prime}=-1,\end{cases}
\end{aligned}
$$

where $k \in \mathbb{Z}, \quad \lambda_{k}^{\prime}(t), \quad s_{0}^{\prime}(t), \quad l_{0}^{\prime}(t), \quad m_{0}^{\prime}(t), \quad n_{0}^{\prime}(t) \in R \backslash\{0\}$, $\lambda_{0}^{\prime}:=\lambda_{0}^{\prime}(t) \in\{ \pm 1\}$, and $\lambda_{k_{1}+k_{2}}(t)=\lambda_{0}^{\prime} \lambda_{k_{1}}^{\prime}(t) \lambda_{k_{2}}{ }^{\prime}(t)$. Furthermore, it is clear that $\theta^{-1}\left(t^{n}, 0\right) \theta \in \operatorname{Ctd}_{\mathbb{C}}(L)$ and $\pi\left(\theta^{-1}\left(t^{n}, 0\right) \theta\right)=\lambda_{0}^{\prime} \lambda_{n}^{\prime}(t)$. Then,

$$
\left(\theta^{-1}\right)_{c}\left(t^{n}\right)=\lambda_{0}^{\prime} \lambda_{n}^{\prime}(t)
$$

Thus, we have $\theta^{-1}\left(c \otimes t^{k}\right)=\left(\theta^{-1}\right)_{c}\left(t^{k}\right) \theta^{-1}\left(c \otimes t^{0}\right)$. For any $k \in \mathbb{Z}$, we have

$$
\begin{aligned}
& c \otimes t^{k}=\theta \theta^{-1}\left(c \otimes t^{k}\right)=\theta\left[\left(\theta^{-1}\right)_{c}\left(t^{k}\right) \theta^{-1}\left(c \otimes t^{0}\right)\right] \\
& = \begin{cases}\theta\left[c \otimes\left(\theta^{-1}\right)_{c}\left(t^{k}\right) s_{0}^{\prime}(t) l_{0}^{\prime}(t)\right], & \text { if } \lambda_{0}^{\prime}=1, \\
-\theta\left[c \otimes\left(\theta^{-1}\right)_{c}\left(t^{k}\right) m_{0}^{\prime}(t) n_{0}^{\prime}(t)\right], & \text { if } \lambda_{0}^{\prime}=-1,\end{cases} \\
& = \begin{cases}\theta_{c}\left[\left(\theta^{-1}\right)_{c}\left(t^{k}\right) s_{0}^{\prime}(t) l_{0}^{\prime}(t)\right] \theta\left(c \otimes t^{0}\right), & \text { if } \lambda_{0}^{\prime}=1, \\
-\theta_{c}\left[\left(\theta^{-1}\right)_{c}\left(t^{k}\right) m_{0}^{\prime}(t) n_{0}^{\prime}(t)\right] \theta\left(c \otimes t^{0}\right), & \text { if } \lambda_{0}^{\prime}=-1,\end{cases} \\
& = \begin{cases}\theta_{c}\left[\left(\theta^{-1}\right)_{c}\left(t^{k}\right)\right] \cdot \theta_{c}\left[s_{0}^{\prime}(t) l_{0}^{\prime}(t)\right] \theta\left(c \otimes t^{0}\right), & \text { if } \lambda_{0}^{\prime}=1, \\
-\theta_{c}\left[\left(\theta^{-1}\right)_{c}\left(t^{k}\right)\right] \cdot \theta_{c}\left[m_{0}^{\prime}(t) n_{0}^{\prime}(t)\right] \theta\left(c \otimes t^{0}\right), & \text { if } \lambda_{0}^{\prime}=-1,\end{cases} \\
& =\theta_{c}\left[\left(\theta^{-1}\right)_{c}\left(t^{k}\right)\right]\left(c \otimes t^{0}\right) \text {. }
\end{aligned}
$$

So, we deduce that $\theta_{c}\left[\left(\theta^{-1}\right)_{c}\left(t^{k}\right)\right]=t^{k}$. Similarly, we have $\left(\theta^{-1}\right)_{c}\left[\theta_{c}\left(t^{k}\right)\right]=t^{k}$ for any $k \in \mathbb{Z}$. Therefore, $\left(\theta_{c}\right)^{-1}=$ $\left(\theta^{-1}\right)_{c}$ and $\theta_{c} \in \operatorname{Aut}_{\mathbb{C}}(R)$.

Theorem 4. For the linear map $\wedge: A u t_{\mathbb{C}}(L) \longrightarrow A u t_{\mathbb{C}}(R)$ with $\theta \mapsto \theta_{c}$, we have

(1) $\wedge$ is a group homomorphism

(2) $\wedge$ is surjective
(3) $\operatorname{Ker} \wedge \cong A u t_{R}(L) \ltimes(\mathbb{C} \otimes F(R))$, where $F(R):=\{\tau \in$ End $_{\mathbb{C}}(R) \mid \tau\left(t^{k}\right)=\delta_{k}(t)-t^{k} \delta_{0}(t)$ for any $\left.k \in \mathbb{Z}\right\}$ and End $_{\mathbb{C}}(R)$ is the set of all linear transformations of $R$

Proof

(1) Let $\theta_{i} \in \operatorname{Aut}_{\mathbb{C}}(L)$ and $\lambda_{i 0}=1, i=1,2$. By (62), we can assume that

$\theta_{i}\left(c \otimes t^{n}\right)=\lambda_{\text {in }}(t) s_{i 0}(t) l_{i 0}(t)\left(c \otimes t^{0}\right)=\lambda_{\text {in }}(t) \theta_{i}\left(c \otimes t^{0}\right), \quad i=1,2$.

By Lemma 7(2), we have

$$
\left(\theta_{i}\right)_{c}\left(t^{n}\right)=\lambda_{\text {in }}(t), \quad i=1,2 .
$$

Then, for any $n \in \mathbb{Z}$,

$$
\begin{aligned}
\theta_{1} \theta_{2}\left(c \otimes t^{n}\right) & =\theta_{1}\left[\left(\theta_{2}\right)_{c}\left(t^{n}\right) \theta_{2}\left(c \otimes t^{0}\right)\right] \\
& =\theta_{1}\left[c \otimes\left(\theta_{2}\right)_{c}\left(t^{n}\right) s_{20}(t) l_{20}(t)\right] \\
& =\left(\theta_{1}\right)_{c}\left[\left(\theta_{2}\right)_{c}\left(t^{n}\right) s_{20}(t) l_{20}(t)\right] \theta_{1}\left(c \otimes t^{0}\right) \\
& =\left(\theta_{1}\right)_{c}\left[\left(\theta_{2}\right)_{c}\left(t^{n}\right)\right]\left(\theta_{1}\right)_{c}\left[s_{20}(t) l_{20}(t)\right] \theta_{1}\left(c \otimes t^{0}\right) \\
& =\left(\theta_{1}\right)_{c}\left(\theta_{2}\right)_{c}\left(t^{n}\right)\left(\theta_{1} \theta_{2}\right)\left(c \otimes t^{0}\right) .
\end{aligned}
$$

Thus,

$\left(\theta_{1} \theta_{2}\right)_{c}\left(t^{n}\right)=\left(\theta_{1}\right)_{c}\left(\theta_{2}\right)_{c}\left(t^{n}\right), \quad$ for any $n \in \mathbb{Z}$.

So, $\left(\theta_{1} \theta_{2}\right)_{c}=\left(\theta_{1}\right)_{c}\left(\theta_{2}\right)_{c}$. The assertion can be similarly checked for other cases besides $\lambda_{i 0}=1$, $i=1$, 2. Then, (1) holds.

(2) Since $\operatorname{Aut}_{\mathbb{C}}(R) \cong \mathbb{C}^{*} \rtimes \mathbb{Z} / 2 \mathbb{Z}$, we have $\tau \in \mathbb{C}^{*}$ or $\tau \in \mathbb{Z} / 2 \mathbb{Z}$ for any $\tau \in \operatorname{Aut}_{\mathbb{C}}(R)$. If $\tau \in \mathbb{C}^{*}$, we may 
assume $\tau\left(t^{n}\right)=t^{n}$ for any $n \in \mathbb{Z}$. By Lemma 6 , let $\theta \in \operatorname{Aut}_{\mathbb{C}}(L)$ such that

$$
\begin{aligned}
& \theta\left(x \otimes t^{n}\right)=t^{n} \theta\left(x \otimes t^{0}\right), \quad x \in\{a, b, c\} \\
& \theta\left(d \otimes t^{n}\right)=t^{n} \theta\left(d \otimes t^{0}\right)+\left(\delta_{n}(t)-t^{n} \delta_{0}(t)\right)\left(c \otimes t^{0}\right) .
\end{aligned}
$$

Then, $\theta_{c}\left(t^{n}\right)=t^{n}=\tau\left(t^{n}\right)$ for any $n \in \mathbb{Z}$. Thus, $\theta_{c}=\tau$. If $\tau$ is the generator of $\mathbb{Z} / 2 \mathbb{Z}$, we assume $\tau(t)=t^{-1}$. Let $\theta \in \operatorname{Aut}_{\mathbb{C}}(L)$ such that

$$
\begin{aligned}
& \theta\left(x \otimes t^{n}\right)=t^{-n} \theta\left(x \otimes t^{0}\right), \quad x \in\{a, b, c\}, \\
& \theta\left(d \otimes t^{n}\right)=t^{-n} \theta\left(d \otimes t^{0}\right)+\left(\delta_{n}(t)-t^{-n} \delta_{0}(t)\right)\left(c \otimes t^{0}\right) .
\end{aligned}
$$

So, $\theta_{c}\left(t^{n}\right)=t^{-n}=\tau\left(t^{n}\right)$ for any $n \in \mathbb{Z}$ and then $\theta_{c}=\tau$. Therefore, $\wedge$ is surjective.

(3) Let $\theta \in \operatorname{Ker} \wedge$. Then, $\theta_{c}=\mathrm{id}_{R}$ and $t^{k}=\theta_{c}\left(t^{k}\right)=$ $\lambda_{0} \lambda_{k}(t)$ for any $k \in \mathbb{Z}$. Thus, by Lemma 6 , we have

$$
\begin{aligned}
& \theta\left(x \otimes t^{k}\right)=t^{k} \theta\left(x \otimes t^{0}\right), \quad x \in\{a, b, c\}, \\
& \theta\left(d \otimes t^{k}\right)=t^{k} \theta\left(d \otimes t^{0}\right)+\left(\delta_{k}(t)-t^{k} \delta_{0}(t)\right)\left(c \otimes t^{0}\right) .
\end{aligned}
$$

If $\delta_{k}(t)-t^{k} \delta_{0}(t)=0$ for any $k \in \mathbb{Z}$, then $\theta \in \operatorname{Aut}_{R}(L)$. If $\delta_{k}(t)-t^{k} \delta_{0}(t) \neq 0, \quad$ define $\varphi \otimes \tau \in \operatorname{Hom}\left(H_{4} /\left[H_{4}, H_{4}\right]\right.$, $\left.Z\left(H_{4}\right)\right) \otimes F(R)$ by

$$
(\varphi \otimes \tau)\left(d \otimes t^{k}\right)=\varphi(d) \otimes \tau\left(t^{k}\right)=c \otimes\left(\delta_{k}(t)-t^{k} \delta_{0}(t)\right), \quad \text { for any } k \in \mathbb{Z}
$$

where $\varphi(d)=c, \quad(\varphi \otimes \tau)\left(d \otimes t^{k}\right)=0$ if $\varphi(d)=0, F(R):=$ $\left\{\tau \in \operatorname{End}_{\mathbb{C}}(R) \mid \tau\left(t^{k}\right)=\delta_{k}(t)-t^{k} \delta_{0}(t)\right.$, for any $\left.k \in \mathbb{Z}\right\}$, and $\operatorname{End}_{\mathbb{C}}(R)$ is the set of all linear transformations of $R$.

Let $\widetilde{\theta}_{i} \in \operatorname{Aut}_{R}(L)$. By (62)-(63), we can assume that $\tilde{\theta}_{i}\left(d \otimes t^{0}\right)=\lambda_{i 0}\left(d \otimes t^{0}\right)+\mu_{i 0}^{1}(t) a \otimes t^{0}+\mu_{i 0}^{2}(t) b \otimes t^{0}+\delta_{i 0}(t) c \otimes t^{0}$,

for $\quad \lambda_{i 0} \in\{ \pm 1\}, \quad i=1,2$. Define the $\operatorname{Aut}_{R}(L) \ltimes\left(\operatorname{Hom}\left(H_{4} /\left[H_{4}, H_{4}\right], Z\left(H_{4}\right)\right) \otimes F(R)\right)$, multiplication is given by

$\left(\widetilde{\theta}_{1}, \varphi_{1} \otimes \tau_{1}\right)\left(\widetilde{\theta}_{2}, \varphi_{2} \otimes \tau_{2}\right)=\left(\widetilde{\theta}_{1} \widetilde{\theta}_{2}, \lambda_{20} \varphi_{1} \otimes \tau_{1}+\widetilde{\theta}_{1}\left(\varphi_{2} \otimes \tau_{2}\right)\right)$.

Furthermore,

$$
\begin{aligned}
(\tilde{\theta}, \varphi \otimes \tau)(1,0) & =(\widetilde{\theta}, \varphi \otimes \tau), \\
(\widetilde{\theta}, \varphi \otimes \tau)^{-1} & =\left(\widetilde{\theta}^{-1},-\lambda_{0} \widetilde{\theta}^{-1}(\varphi \otimes \tau)\right), \\
(\tilde{\theta}, 0)(1, \varphi \otimes \tau)(\tilde{\theta}, 0)^{-1} & =\left(1, \lambda_{0} \tilde{\theta}(\varphi \otimes \tau)\right),
\end{aligned}
$$

where $\left(\tilde{\theta}_{i}, \varphi_{i} \otimes \tau_{i}\right),(\widetilde{\theta}, \varphi \otimes \tau) \in \operatorname{Aut}_{R}(L) \ltimes\left(\operatorname{Hom}\left(H_{4} /\left[H_{4}\right.\right.\right.$, $\left.\left.\left.H_{4}\right], Z\left(H_{4}\right)\right) \otimes F(R)\right), \lambda_{i 0}, \lambda_{0} \in\{ \pm 1\}, i=1,2$.

Define a map $\eta$ : Ker $\wedge \longrightarrow \operatorname{Aut}_{R}(L) \ltimes\left(\operatorname{Hom}\left(H_{4} /\right.\right.$ $\left.\left.\left[H_{4}, H_{4}\right], Z\left(H_{4}\right)\right) \otimes F(R)\right)$ by

$$
\theta \mapsto(\tilde{\theta}, \varphi \otimes \tau),
$$

for $\tilde{\theta} \in \operatorname{Aut}_{R}(L), \varphi \otimes \tau \in \operatorname{Hom}\left(H_{4} /\left[H_{4}, H_{4}\right], Z\left(H_{4}\right)\right) \otimes F$ $(R)$, and

$$
\begin{aligned}
& (\tilde{\theta}, \varphi \otimes \tau)\left(x \otimes t^{k}\right)=\tilde{\theta}\left(x \otimes t^{k}\right), \\
& (\tilde{\theta}, \varphi \otimes \tau)\left(d \otimes t^{k}\right)=\tilde{\theta}\left(d \otimes t^{k}\right)+(\varphi \otimes \tau)\left(d \otimes t^{k}\right),
\end{aligned}
$$

for $x \in\{a, b, c\}$ and any $k \in \mathbb{Z}$. It is easy to see that $\eta$ is welldefined and bijective. Let $\theta_{i} \in \operatorname{Ker} \wedge$ and $\eta\left(\theta_{i}\right)=\left(\widetilde{\theta}_{i}, \varphi_{i} \otimes \tau_{i}\right)$, $i=1,2$. By $(80)-(86)$, we have

$$
\begin{aligned}
\theta_{1} \theta_{2}\left(d \otimes t^{k}\right)= & \theta_{1}\left[t^{k} \widetilde{\theta}_{2}\left(d \otimes t^{0}\right)+\left(\varphi_{2} \otimes \tau_{2}\right)\left(d \otimes t^{k}\right)\right] \\
= & \theta_{1}\left[\lambda_{20} d \otimes t^{k}+\mu_{20}^{1}(t) a \otimes t^{k}+\mu_{20}^{2}(t) b \otimes t^{k}+\delta_{20}(t) c \otimes t^{k}\right]+\widetilde{\theta}_{1}\left(\varphi_{2} \otimes \tau_{2}\right)\left(d \otimes t^{k}\right) \\
= & t^{k} \lambda_{20} \tilde{\theta}_{1}\left(d \otimes t^{0}\right)+\lambda_{20}\left(\varphi_{1} \otimes \tau_{1}\right)\left(d \otimes t^{k}\right)+t^{k} \mu_{20}^{1}(t) \widetilde{\theta}_{1}\left(a \otimes t^{0}\right)+t^{k} \mu_{20}^{2}(t) \widetilde{\theta}_{1}\left(b \otimes t^{0}\right) \\
& +t^{k} \delta_{20}(t) \tilde{\theta}_{1}\left(c \otimes t^{0}\right)+\widetilde{\theta}_{1}\left(\varphi_{2} \otimes \tau_{2}\right)\left(d \otimes t^{k}\right) \\
= & t^{k} \widetilde{\theta}_{1} \widetilde{\theta}_{2}\left(d \otimes t^{0}\right)+\left(\lambda_{20} \varphi_{1} \otimes \tau_{1}+\widetilde{\theta}_{1}\left(\varphi_{2} \otimes \tau_{2}\right)\right)\left(d \otimes t^{k}\right) .
\end{aligned}
$$


Then, $\eta\left(\theta_{1} \theta_{2}\right)=\left(\tilde{\theta}_{1} \tilde{\theta}_{2}, \lambda_{20} \varphi_{1} \otimes \tau_{1}+\tilde{\theta}_{1}\left(\varphi_{2} \otimes \tau_{2}\right)\right)=\eta\left(\theta_{1}\right)$ $\eta\left(\theta_{2}\right)$. Thus, $\eta$ is a group isomorphism. Furthermore, since $H_{4} /\left[H_{4}, H_{4}\right]=\mathbb{C} d$ and $Z\left(H_{4}\right)=\mathbb{C} c$, it is clear that $\operatorname{Hom}\left(\mathrm{H}_{4} /\left[\mathrm{H}_{4}, \mathrm{H}_{4}\right], Z\left(\mathrm{H}_{4}\right)\right)$ is isomorphic to $\mathbb{C}$ as vector spaces over $\mathbb{C}$. Then, (3) is proved.

\section{Corollary 4}

$$
\begin{aligned}
\operatorname{Aut}_{\mathbb{C}}(L) & \cong \operatorname{Aut}_{\mathbb{C}}(R) \ltimes \operatorname{Ker} \wedge \\
& \cong \operatorname{Aut}_{\mathbb{C}}(R) \ltimes\left(A u t_{R}(L) \ltimes(\mathbb{C} \otimes F(R))\right) .
\end{aligned}
$$

Proof. It follows from Theorem 4.

\section{The Derivation Algebra $\operatorname{Der}_{\mathfrak{C}}(L)$ of $L$}

In this section, we study the derivations of $L=H_{4} \otimes_{\mathbb{C}} R$, where $R=\mathbb{C}\left[t^{ \pm 1}\right]$. Denote by $\operatorname{Der}_{R}(L)$ the algebra of all $R$-linear derivations of $L$.

Lemma 8. Let $g \in \operatorname{Der}\left(H_{4}\right)$. Then,

$$
\begin{aligned}
& g(a)=\lambda_{1} a+\delta_{1} c, \\
& g(b)=\mu_{2} b+\delta_{2} c, \\
& g(c)=\left(\lambda_{1}+\mu_{2}\right) c, \\
& g(d)=-\delta_{2} a-\delta_{1} b+\delta_{3} c,
\end{aligned}
$$

where $\lambda_{1}, \mu_{2}, \delta_{i} \in \mathbb{C}, 1 \leq i \leq 3$.

Proof. For $g \in \operatorname{Der}\left(H_{4}\right)$, we can assume that

$$
\begin{aligned}
& g(a)=\lambda_{1} a+\mu_{1} b+\delta_{1} c, \\
& g(b)=\lambda_{2} a+\mu_{2} b+\delta_{2} c, \\
& g(d)=\alpha(d)+\lambda_{3} a+\mu_{3} b+\delta_{3} c,
\end{aligned}
$$

where $\lambda_{i}, \mu_{i}, \delta_{i}, \alpha \in \mathbb{C}, 1 \leq i \leq 3$. Since

$$
\begin{aligned}
g(a) & =[g(d), a]+[d, g(a)], \\
-g(b) & =[g(d), b]+[d, g(b)], \\
g(c) & =[g(a), b]+[a, g(b)],
\end{aligned}
$$

we have

$$
\begin{aligned}
\alpha & =0, \\
\mu_{1} & =0, \\
\lambda_{2} & =0, \\
\mu_{3} & =-\delta_{1}, \\
\lambda_{3} & =-\delta_{2} .
\end{aligned}
$$

Thus, the lemma is proved.

Let $d_{i} \in \operatorname{Der}\left(H_{4}\right)(1 \leq i \leq 3)$ be such that

$$
\begin{aligned}
& d_{1}(a)=a, \\
& d_{1}(c)=c, \\
& d_{1}(b)=d_{1}(d)=0, \\
& d_{2}(b)=b, \\
& d_{2}(c)=c, \\
& d_{2}(a)=d_{2}(d)=0, \\
& d_{3}(d)=c, \\
& d_{3}(a)=d_{3}(b)=d_{3}(c)=0 .
\end{aligned}
$$

\section{Theorem}

5. $\operatorname{Der}\left(H_{4}\right)=\mathbb{C} d_{1} \oplus \mathbb{C} d_{2} \oplus \mathbb{C} d_{3} \oplus \mathbb{C a d}(a) \oplus \mathbb{C a d}(b)$.

Proof. For any $g \in \operatorname{Der}\left(H_{4}\right)$, by Lemma 8 , we have

$$
g=\lambda_{1} d_{1}+\mu_{2} d_{2}+\delta_{3} d_{3}+\delta_{2} \operatorname{ad}(a)-\delta_{1} \operatorname{ad}(b) .
$$

It is clear that $d_{1}, d_{2}, d_{3}, \operatorname{ad}(a)$, and $\operatorname{ad}(b)$ are linearly independent.

Lemma 9. Let $D \in \operatorname{Der}_{\mathbb{C}}(L)$. Then,

$$
\begin{aligned}
& D\left(x \otimes t^{n}\right)=x \otimes\left(\lambda_{1 n}(t)-\lambda_{10}(t) t^{n}\right)+t^{n} D\left(x \otimes t^{0}\right), \\
& D\left(d \otimes t^{n}\right)=d \otimes\left(\lambda_{1 n}(t)-\lambda_{10}(t) t^{n}\right)+t^{n} D\left(d \otimes t^{0}\right)+c \otimes\left(\delta_{3 n}(t)-\delta_{30}(t) t^{n}\right),
\end{aligned}
$$

where $x \in\{a, b, c\}, \quad \lambda_{1 n}(t), \quad \delta_{3 n}(t) \in R$, and $\lambda_{1 m+n}(t)=\quad$ Proof. For $D \in \operatorname{Der}_{\mathbb{C}}(L)$, we may assume that $\lambda_{1 n}(t) t^{m}+\lambda_{1 m}(t) t^{n}-\lambda_{10}(t) t^{m+n}, m, n \in \mathbb{Z}$.

$$
\begin{aligned}
& D\left(a \otimes t^{n}\right)=\lambda_{1 n}(t)\left(a \otimes t^{0}\right)+\mu_{1 n}(t)\left(b \otimes t^{0}\right)+\delta_{1 n}(t)\left(c \otimes t^{0}\right), \\
& D\left(b \otimes t^{n}\right)=\lambda_{2 n}(t)\left(a \otimes t^{0}\right)+\mu_{2 n}(t)\left(b \otimes t^{0}\right)+\delta_{2 n}(t)\left(c \otimes t^{0}\right), \\
& D\left(d \otimes t^{n}\right)=\alpha_{n}(t)\left(d \otimes t^{0}\right)+\lambda_{3 n}(t)\left(a \otimes t^{0}\right)+\mu_{3 n}(t)\left(b \otimes t^{0}\right)+\delta_{3 n}(t)\left(c \otimes t^{0}\right),
\end{aligned}
$$


where $\lambda_{\text {in }}(t), \mu_{\text {in }}(t), \delta_{\text {in }}(t), \alpha_{n}(t) \in R, i \in\{1,2,3\}$. By

$$
\begin{aligned}
D\left(a \otimes t^{n}\right) & =\left[D(d \otimes 1), a \otimes t^{n}\right]+\left[d \otimes 1, D\left(a \otimes t^{n}\right)\right]=\left[D\left(d \otimes t^{n}\right), a \otimes 1\right]+\left[d \otimes t^{n}, D(a \otimes 1)\right], \\
-D\left(b \otimes t^{n}\right) & =\left[D(d \otimes 1), b \otimes t^{n}\right]+\left[d \otimes 1, D\left(b \otimes t^{n}\right)\right]=\left[D\left(d \otimes t^{n}\right), b \otimes 1\right]+\left[d \otimes t^{n}, D(b \otimes 1)\right], \\
D\left(c \otimes t^{n}\right) & =\left[D(a \otimes 1), b \otimes t^{n}\right]+\left[a \otimes 1, D\left(b \otimes t^{n}\right)\right]=\left[D\left(a \otimes t^{n}\right), b \otimes 1\right]+\left[a \otimes t^{n}, D(b \otimes 1)\right],
\end{aligned}
$$

we have

$$
\begin{aligned}
\alpha_{0}(t) & =0 \\
\mu_{1 n}(t) & =0 \\
\lambda_{2 n}(t) & =0 \\
\delta_{1 n}(t) & =\delta_{10}(t) t^{n} \\
\delta_{2 n}(t) & =\delta_{20}(t) t^{n} \\
\alpha_{n}(t) & =\lambda_{1 n}(t)-\lambda_{10}(t) t^{n}=\mu_{2 n}(t)-\mu_{20}(t) t^{n} \\
\mu_{3 n}(t) & =-\delta_{1 n}(t) \\
\lambda_{3 n}(t) & =-\delta_{2 n}(t)
\end{aligned}
$$

\section{Thus,}

$$
\begin{aligned}
D\left(a \otimes t^{n}\right) & =\lambda_{1 n}(t)\left(a \otimes t^{0}\right)+\delta_{10}(t) t^{n}\left(c \otimes t^{0}\right)=a \otimes\left(\lambda_{1 n}(t)-\lambda_{10}(t) t^{n}\right)+t^{n} D\left(a \otimes t^{0}\right), \\
D\left(b \otimes t^{n}\right) & =\mu_{2 n}(t)\left(b \otimes t^{0}\right)+\delta_{20}(t) t^{n}\left(c \otimes t^{0}\right)=b \otimes\left(\lambda_{1 n}(t)-\lambda_{10}(t) t^{n}\right)+t^{n} D\left(b \otimes t^{0}\right), \\
D\left(c \otimes t^{n}\right) & =\left(\lambda_{1 n}(t)+\mu_{20}(t) t^{n}\right)\left(c \otimes t^{0}\right)=c \otimes\left(\lambda_{1 n}(t)-\lambda_{10}(t) t^{n}\right)+t^{n} D\left(c \otimes t^{0}\right), \\
D\left(d \otimes t^{n}\right) & =\left(\lambda_{1 n}(t)-\lambda_{10}(t) t^{n}\right)\left(d \otimes t^{0}\right)-\delta_{20}(t) t^{n}\left(a \otimes t^{0}\right)-\delta_{10}(t) t^{n}\left(b \otimes t^{0}\right)+\delta_{3 n}(t)\left(c \otimes t^{0}\right) \\
& =d \otimes\left(\lambda_{1 n}(t)-\lambda_{10}(t) t^{n}\right)+t^{n} D\left(d \otimes t^{0}\right)+c \otimes\left(\delta_{3 n}(t)-\delta_{30}(t) t^{n}\right) . \\
\rho(D)\left(t^{n}\right) & =\lambda_{1 n}(t)-\lambda_{10}(t) t^{n} \Leftrightarrow \pi\left(\left[D,\left(t^{n}, 0\right)\right]\right) \\
& =\lambda_{1 n}(t)-\lambda_{10}(t) t^{n} .
\end{aligned}
$$

Since

$$
\begin{aligned}
D\left(a \otimes t^{n+m}\right)= & \lambda_{1 m+n}(t)\left(a \otimes t^{0}\right)+\delta_{10}(t) t^{m+n}\left(c \otimes t^{0}\right) \\
= & {\left[D\left(d \otimes t^{n}\right), a \otimes t^{m}\right]+\left[d \otimes t^{n}, D\left(a \otimes t^{m}\right)\right] } \\
= & a \otimes\left(\lambda_{1 n}(t) t^{m}+\lambda_{1 m}(t) t^{n}-\lambda_{10}(t) t^{m+n}\right) \\
& +c \otimes \delta_{10}(t) t^{m+n},
\end{aligned}
$$

we have $\lambda_{1 m+n}(t)=\lambda_{1 n}(t) t^{m}+\lambda_{1 m}(t) t^{n}-\lambda_{10}(t) t^{m+n}$. The lemma is proved.

\section{Theorem 6}

(i) Let $D \in \operatorname{Der}_{\mathbb{C}}(L)$. Then, $\left[D,\left(t^{n}, 0\right)\right] \in C t d_{\mathbb{C}}(L)$ and $\pi\left(\left[D,\left(t^{n}, 0\right)\right]\right)=\lambda_{1 n}(t)-\lambda_{10}(t) t^{n}$, where $\pi$ is defined in (65).

(ii) Given $D \in \operatorname{Der}_{\mathbb{C}}(L)$. The linear map $\rho: \operatorname{Der}_{\mathbb{C}}(L)$ $\longrightarrow \operatorname{Der}_{\mathbb{C}}(R)$ is defined by the following identity:
Then,

(1) $\rho$ is a Lie algebra homomorphism

(2) $\rho$ is surjective

(3) $\operatorname{Ker}(\rho) \cong \operatorname{Der}_{R}(L)+\mathbb{C} \otimes E(R)$, where $E(R):=\{\tau \in$ $E n d_{\mathbb{C}}(R) \mid \tau\left(t^{n}\right)=\delta_{3 n}(t)-\delta_{30}(t) t^{n}$, for $\delta_{3 n}(t) \in R$ and any $n \in \mathbb{Z}\}, \operatorname{End}_{\mathbb{C}}(R)$ is the set of all linear transformations of $R$

Proof

(i) For any $X, Y \in L$, we have

$$
\begin{aligned}
{\left[D,\left(t^{n}, 0\right)\right]([X, Y]) } & =D\left(t^{n}, 0\right)([X, Y])-\left(t^{n}, 0\right) D([X, Y]) \\
& =D\left(\left[t^{n} X, Y\right]\right)-\left(t^{n}, 0\right)([D(X), Y]+[X, D(Y)]) \\
& =\left[D\left(t^{n} X\right), Y\right]-\left[t^{n} D(X), Y\right]=\left[\left[D,\left(t^{n}, 0\right)\right](X), Y\right] .
\end{aligned}
$$


Then, $\left[D,\left(t^{n}, 0\right)\right] \in \operatorname{Ctd}_{\mathbb{C}}(L)$. By Lemma 9, for $x \in\{a, b, c\}$ and $m \in \mathbb{Z}$, we have

$$
\begin{aligned}
{\left[D,\left(t^{n}, 0\right)\right]\left(x \otimes t^{m}\right) } & =D\left(x \otimes t^{n+m}\right)-\left(t^{n}, 0\right) D\left(x \otimes t^{m}\right) \\
& =x \otimes\left(\lambda_{1 n+m}(t)-\lambda_{1 m}(t) t^{n}\right) \\
& =\left(\lambda_{1 n}(t)-\lambda_{10}(t) t^{n}\right)\left(x \otimes t^{m}\right), \\
{\left[D,\left(t^{n}, 0\right)\right]\left(d \otimes t^{m}\right) } & =D\left(d \otimes t^{n+m}\right)-\left(t^{n}, 0\right) D\left(d \otimes t^{m}\right) \\
& =d \otimes\left(\lambda_{1 n+m}(t)-\lambda_{1 m}(t) t^{n}\right)+c \otimes\left(\delta_{3 n+m}(t)-\delta_{3 m}(t) t^{n}\right) \\
& =\left(\lambda_{1 n}(t)-\lambda_{10}(t) t^{n}\right)\left(d \otimes t^{m}\right)+\left(\delta_{3 n+m}(t)-\delta_{3 m}(t) t^{n}\right)\left(c \otimes t^{0}\right) .
\end{aligned}
$$

Therefore, by Theorem 3 and the definition of $\pi$ in (65), we have $\pi\left(\left[D,\left(t^{n}, 0\right)\right]\right)=\lambda_{1 n}(t)-\lambda_{10}(t) t^{n}$.

(ii) By (i) and (101), it is easy to see that $\rho(D)$ is a linear transformation of $R$. For any $n, m \in \mathbb{Z}$, we have

$$
\begin{aligned}
\rho(D)\left(t^{n+m}\right) & =\lambda_{1 n+m}(t)-\lambda_{10}(t) t^{n+m} \\
& =\lambda_{1 n}(t) t^{m}+\lambda_{1 m}(t) t^{n}-2 \lambda_{10}(t) t^{n+m} \\
& =\rho(D)\left(t^{n}\right) t^{m}+t^{n} \rho(D)\left(t^{m}\right) .
\end{aligned}
$$

Then, $\rho(D) \in \operatorname{Der}_{\mathbb{C}}(R)$.

(1) For $D_{i} \in \operatorname{Der}_{\mathbb{C}}(L)$, by (99), we can assume that

$$
D_{i}\left(c \otimes t^{0}\right)=c \otimes\left(\lambda_{10}^{i}(t)+\mu_{20}^{i}(t)\right), \quad i=1,2 .
$$

For any $n \in \mathbb{Z}$, we have

$$
\begin{aligned}
{\left[D_{1}, D_{2}\right]\left(c \otimes t^{n}\right)=} & c \otimes \rho\left(\left[D_{1}, D_{2}\right]\right)\left(t^{n}\right)+t^{n}\left[D_{1}, D_{2}\right]\left(c \otimes t^{0}\right) \\
= & D_{1}\left[c \otimes \rho\left(D_{2}\right)\left(t^{n}\right)+t^{n} D_{2}\left(c \otimes t^{0}\right)\right]-D_{2}\left[c \otimes \rho\left(D_{1}\right)\left(t^{n}\right)+t^{n} D_{1}\left(c \otimes t^{0}\right)\right] \\
= & c \otimes \rho\left(D_{1}\right) \rho\left(D_{2}\right)\left(t^{n}\right)+\rho\left(D_{2}\right)\left(t^{n}\right) D_{1}\left(c \otimes t^{0}\right)+D_{1}\left[t^{n} D_{2}\left(c \otimes t^{0}\right)\right] \\
& -c \otimes \rho\left(D_{2}\right) \rho\left(D_{1}\right)\left(t^{n}\right)-\rho\left(D_{1}\right)\left(t^{n}\right) D_{2}\left(c \otimes t^{0}\right)-D_{2}\left[t^{n} D_{1}\left(c \otimes t^{0}\right)\right] \\
= & c \otimes\left[\rho\left(D_{1}\right), \rho\left(D_{2}\right)\right]\left(t^{n}\right)+t^{n} c \otimes \rho\left(D_{1}\right)\left(\lambda_{10}^{2}(t)+\mu_{20}^{2}(t)\right)-t^{n} c \otimes \rho\left(D_{2}\right)\left(\lambda_{10}^{1}(t)+\mu_{20}^{1}(t)\right) \\
= & c \otimes\left[\rho\left(D_{1}\right), \rho\left(D_{2}\right)\right]\left(t^{n}\right)+t^{n}\left[D_{1}, D_{2}\right]\left(c \otimes t^{0}\right) .
\end{aligned}
$$

Thus, $\rho\left(\left[D_{1}, D_{2}\right]\right)=\left[\rho\left(D_{1}\right), \rho\left(D_{2}\right)\right]$.

(2) Since $\operatorname{Der}_{\mathbb{C}}(R)=\operatorname{Der}_{\mathbb{C}}\left(\mathbb{C}\left[t^{ \pm 1}\right]\right)$ is a Witt algebra and $\operatorname{Der}_{\mathbb{C}}(R)=\operatorname{Span}_{\mathbb{C}}\left\{\delta_{i}=-t^{i+1}(\mathrm{~d} / \mathrm{d} t) \mid i \in \mathbb{Z}\right\}$, for any $\delta_{i}=-t^{i+1}(\mathrm{~d} / \mathrm{d} t) \in \operatorname{Der}_{\mathbb{C}}(R), i \in \mathbb{Z}$, there exists $D_{i}=$ $\mathrm{id}_{H_{4}} \otimes \delta_{i} \in \operatorname{Der}_{\mathbb{C}}(L)$ such that $\rho\left(D_{i}\right)=\delta_{i}$.

(3) For any $D \in \operatorname{Ker}(\rho)$, we have $\rho(D)=0$ and by Lemma 9,

$$
\begin{aligned}
& D\left(x \otimes t^{n}\right)=t^{n} D\left(x \otimes t^{0}\right), \quad x \in\{a, b, c\}, \\
& D\left(d \otimes t^{n}\right)=t^{n} D\left(d \otimes t^{0}\right)+c \otimes\left(\delta_{3 n}(t)-\delta_{30}(t) t^{n}\right) .
\end{aligned}
$$

If $\delta_{3 n}(t)-\delta_{30}(t) t^{n}=0$ for any $n \in \mathbb{Z}$, then $D \in \operatorname{Der}_{R}(L)$. If $\delta_{3 n}(t)-\delta_{30}(t) t^{n} \neq 0$, define $\varphi \otimes \tau \in \operatorname{Hom}\left(H_{4} /\left[H_{4}\right.\right.$, $\left.\left.H_{4}\right], Z\left(H_{4}\right)\right) \otimes E(R)$ by

$$
(\varphi \otimes \tau)\left(d \otimes t^{n}\right)=\varphi(d) \otimes \tau\left(t^{n}\right)=c \otimes\left(\delta_{3 n}(t)-\delta_{30}(t) t^{n}\right),
$$

where $\varphi(d)=c, \quad(\varphi \otimes \tau)\left(d \otimes t^{n}\right)=0$ if $\varphi(d)=0, E(R):=$ $\left\{\tau \in \operatorname{End}_{\mathbb{C}}(R) \mid \tau\left(t^{n}\right)=\delta_{3 n}(t)-\delta_{30}(t) t^{n}, \quad\right.$ for $\delta_{3 n}(t) \in R$ and any $n \in \mathbb{Z}\}$, $\operatorname{End}_{\mathbb{C}}(R)$ is the set of all linear transformations of $R$. So, $\operatorname{Ker}(\rho) \subseteq \operatorname{Der}_{R}(L)+\operatorname{Hom}\left(H_{4} /\left[H_{4}\right.\right.$, $\left.\left.H_{4}\right], Z\left(H_{4}\right)\right) \otimes E(R)$. Conversely, for any $D_{R} \in \operatorname{Der}_{R}(L)$, $y \in H_{4}$, we have $D_{R}\left(y \otimes t^{n}\right)=t^{n} D_{R}\left(y \otimes t^{0}\right)$. By Lemma 9 and (101), it follows that $\rho\left(D_{R}\right)=0$ and $\operatorname{Der}_{R}(L) \subseteq \operatorname{Ker}(\rho)$. For any $\varphi \otimes \tau \in \operatorname{Hom}\left(H_{4} /\left[H_{4}, H_{4}\right], Z\left(H_{4}\right)\right) \otimes E(R)$, by the definition of $\varphi \otimes \tau$, it is clear that $\varphi \otimes \tau \in \operatorname{Der}_{\mathbb{C}}(L)$ and $\varphi \otimes \tau \in \operatorname{Ker}(\rho)$. Therefore, $\operatorname{Der}_{R}(L)+\operatorname{Hom}\left(H_{4} /\left[H_{4}, H_{4}\right]\right.$, $\left.Z\left(H_{4}\right)\right) \otimes E(R)=\operatorname{Ker}(\rho)$. Moreover, since $H_{4} /\left[H_{4}, H_{4}\right]=$ $\mathbb{C} d$ and $Z\left(H_{4}\right)=\mathbb{C} c$, it is easy to see that $\operatorname{Hom}\left(H_{4} /\right.$ $\left.\left[H_{4}, H_{4}\right], Z\left(H_{4}\right)\right)$ is isomorphic to $\mathbb{C}$ as vector spaces over $\mathbb{C}$. Thus, (3) is proved. 


\section{Corollary 5}

$$
\begin{aligned}
\operatorname{Der}_{\mathbb{C}}(L) & \cong \operatorname{Der}_{\mathbb{C}}(R)+\operatorname{Ker}(\rho) \\
& \cong i d_{H_{4}} \otimes \operatorname{Der}_{\mathbb{C}}(R)+\operatorname{Der}_{R}(L)+\mathbb{C} \otimes E(R) \\
& \cong \operatorname{Ctd}_{\mathbb{C}}\left(H_{4}\right) \otimes \operatorname{Der}_{\mathbb{C}}(R) \oplus \operatorname{Der}_{\mathbb{C}}\left(H_{4}\right) \otimes R \oplus \mathbb{C} \otimes \frac{E(R)}{\operatorname{Der}_{\mathbb{C}}(R)}
\end{aligned}
$$

Proof. The first and second isomorphism relations follow from Theorem 6 . The third one follows from

$$
\begin{aligned}
\operatorname{Der}_{R}(L) & =\operatorname{Der}_{R}\left(H_{4} \otimes_{\mathbb{C}} R\right) \cong \operatorname{Der}\left(H_{4} \otimes 1, H_{4} \otimes R\right) \\
& =\operatorname{Der}\left(H_{4}\right) \otimes R .
\end{aligned}
$$

See also Theorem 7.1 in [13], Theorem 1.1 in [14], and Lemmas 2.7 and 2.8 in [15]. Furthermore, by Lemma 5, we have

$$
\operatorname{Ctd}_{\mathbb{C}}\left(H_{4}\right) \otimes \operatorname{Der}_{\mathbb{C}}(R)=i d_{H_{4}} \otimes \operatorname{Der}_{\mathbb{C}}(R)+\mathbb{C} \otimes \operatorname{Der}_{\mathbb{C}}(R) .
$$

Then, the corollary is proved.

Remark 2. The last isomorphism relation in Corollary 5 overlaps with Theorem 7.1 in [13], Theorem 1.1 in [14], Corollary 2.2 in [16], Theorem 2.9 in [15], and Theorem 4.2 in [12].

\section{Data Availability}

The data of the Lie algebra relations used to support the findings of this study are included within the article.

\section{Conflicts of Interest}

The author declares that there are no conflicts of interest.

\section{Acknowledgments}

This study was supported by China NSF grants Nos. 11801477 and 11771281.

\section{References}

[1] E. Witten, "Non-abelian bosonization in two dimensions," Communications in Mathematical Physics, vol. 92, no. 4, pp. 455-472, 1984.

[2] E. Kiritsis and C. Kounnas, "String propagation in gravitational wave backgrounds," Physics Letters B, vol. 320, no. 3-4, pp. 264-272, 1994.

[3] C. R. Nappi and E. Witten, "Wess-Zumino-Witten model based on a nonsemisimple group," Physical Review Letters, vol. 71, no. 23, pp. 3751-3753, 1993.

[4] Y. Cheung, L. Freidel, and K. Savvidy, "Strings in gravimagnetic fields," Journal of High Energy Physics, vol. 2, no. 54, pp. 1-47, 2004.
[5] G. D’Appollonio and E. Kiritsis, "String interactions in gravitational wave backgrounds," Nuclear Physics B, vol. 674, pp. 80-170, 2003.

[6] G. D'Appollonio and T. Quella, "The abelian cosets of the Heisenberg group," Journal of High Energy Physics, vol. 11, no. 045, pp. 1-27, 2007.

[7] G. D'Appollonio and T. Quella, "The diagonal cosets of the Heisenberg group," Journal of High Energy Physics, vol. 5, no. 60, pp. 1-41, 2008.

[8] Y. Bao, C. Jiang, and Y. Pei, "Representations of affine NappiWitten algebras," Journal of Algebra, vol. 342, no. 1 , pp. 111-133, 2011.

[9] X. Chen and C. Jiang, "Whittaker modules for the twisted

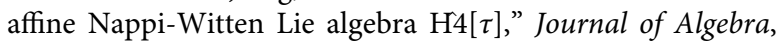
vol. 546, pp. 37-61, 2020.

[10] S. Wang, "Automorphism groups of vertex operator algebras associated with the affine Nappi-Witten algebra $\widehat{H}_{4}$," Acta Mathematica Sinica Chinese Series, vol. 57, no. 2, pp. 331-338, 2014.

[11] B. Allison, S. Berman, and A. Pianzola, "Covering algebras II: isomorphism of loop algebras," Journal für die reine und angewandte Mathematik, vol. 571, pp. 39-71, 2004.

[12] A. Pianzola, "Derivations of certain algebras defined by étale descent," Mathematische Zeitschrift, vol. 264, no. 3, pp. 485-495, 2010.

[13] R. E. Block, "Determination of the differentiably simple rings with a minimal ideal," The Annals of Mathematics, vol. 90, no. 3, pp. 433-459, 1969.

[14] G. M. Benkart and R. V. Moody, "Derivations, central extensions, and affine Lie algebras," Algebras Groups and Geometrics, vol. 3, pp. 456-492, 1986.

[15] S. Azam, "Derivations of tensor product algebras," Communications in Algebra, vol. 36, no. 3, pp. 905-927, 2008.

[16] P. Zusmanovich, "Low-dimensional cohomology of current Lie algebras and analogs of the Riemann tensor for loop manifolds," Linear Algebra and Its Applications, vol. 407, pp. 71-104, 2005. 